\title{
Numerical approximations to the scaled first derivatives of the solution to a two parameter singularly perturbed problem
}

\author{
E. O'Riordan and M. L. Pickett \\ School of Mathematical Sciences, Dublin City University, Dublin 9, Ireland. email: \\ eugene.oriordan@dcu.ie \\ Department of Mathematics, University of Portsmouth, U.K. email: \\ maria.pickett@port.ac.uk
}

\begin{abstract}
A singularly perturbed problem involving two singular perturbation parameters is discretized using the classical upwinded finite difference scheme on an appropriate piecewise-uniform Shishkin mesh. Scaled discrete derivatives (with scaling only used within the layers) are shown to be parameter uniformly convergent to the scaled first derivatives of the continuous solution.
\end{abstract}

Keywords: Singularly perturbed, Two parameter, Scaled first derivative AMS subject classifications: 65L11, 65L12, 65L70.

\section{Introduction}

A characteristic feature of singularly perturbed problems is the appearance of steep gradients in the solution. In order to generate pointwise accurate parameter-uniform [3] numerical approximations to the solution in the layer regions, where the steep gradients occur, it is useful to identify the correct scale of the gradients. In the case of singular perturbation problems involving one perturbation parameter, this scale is normally some inverse power of the singular perturbation parameter. In the case of singular perturbation problems involving two perturbation parameters, the scale of the gradients appearing in the layer regions can depend on one or both singular perturbation parameters. Outside the layer regions, the gradients are of order one. In this paper, we generate pointwise accurate numerical approximations to both the solution and the scaled first derivative of the solution. The first derivative of the solution is unbounded within the layers and so we estimate the accuracy of an appropriately scaled first derivative within the layered regions.

In the case of singularly perturbed boundary value problems of the form

$$
-\varepsilon u^{\prime \prime}+a(x) u^{\prime}(x)+b(x) u=f(x), x \in(0,1) ; \quad a(x), b(x), \varepsilon>0 ;
$$


which contain a single perturbation parameter $0<\varepsilon \leq 1$, parameter-uniform pointwise error bounds [3] on numerical approximations to the scaled first derivative $\varepsilon u^{\prime}$ have been established $[1,2,3]$. In these publications, a scaling factor of $\varepsilon$ is applied (to the error in estimating $u^{\prime}$ ) throughout the domain [0,1]. Kopteva and Stynes [8] established a first order error bound for approximations to the first derivative of the solution, where the scaling was only applied within the computational layer region, where $x_{i} \leq C \varepsilon \ln N$. Shishkin $[15,16]$ examined a more sophisticated metric, which involved the scaling factor smoothly changing from a scale of $\varepsilon$ for $x \leq \varepsilon$ to no scaling outside the analytical layer region, where $x \geq C \varepsilon \ln (1 / \varepsilon)$. However, Shishkin [16] also established that a numerical method combining an upwind finite difference scheme with a piecewise-uniform layeradapted mesh is not a parameter-uniform numerical method in this new metric. In this paper, we will establish parameter-uniform bounds on approximations to the scaled first derivative of the solution of the two parameter singularly perturbed boundary value problem

$$
-\varepsilon u^{\prime \prime}+\mu a(x) u^{\prime}(x)+b(x) u=f(x), x \in(0,1) ; \quad a(x), b(x), \varepsilon>0, \mu \geq 0 ;
$$

where we simply scale (by appropriate factors) within the analytical layer regions only. Our method of proof is based on the analysis in $[5,6,7]$, which dealt with singularly perturbed parabolic and elliptic problems containing a single perturbation parameter.

In [4] a second order parameter-uniform scheme was constructed for the two parameter problem. Using the same scaling (as in the current paper) such a scheme automatically has first order (ignoring logarithmic factors) convergence for the scaled first derivatives. However, the finite difference operator involved in the scheme from [4] is rather complicated. Here, we deal with the simple upwind finite difference operator, which is only a first order scheme for the solution. Nevertheless, we will prove that this simple numerical method generates first order (up to logarithmic factors) approximations to the scaled first derivatives. The key ingredient within the numerical method is the design of a suitable piecewise-uniform Shishkin mesh.

Note that in $[9,10,17]$, the transition parameters for the Shishkin mesh, involve the maximum and minimum values (taken over the range of the independent variable $x \in[0,1])$ of the roots of the quadratic function $-\varepsilon \lambda^{2}(x)+$ $\mu a(x) \lambda(x)+b(x)$, which is non-trivial in the case of variable coefficients. Below the appropriate scaled weighting factors to be used in estimating the derivatives and in the choice of the transition parameters for the mesh are explicitly stated in terms of the two singular perturbation parameters $\varepsilon$ and $\mu$. Note also that the decomposition of the continuous solution derived in $\S 2$ is different to the decomposition used in $[9,10,17]$.

Most of the recent literature on two-parameter singularly perturbed problems (e.g., see $[4,9,10,12]$ and the references therein) deals with parameteruniform $C^{0}$-convergence. In this paper, we focus on parameter-uniform convergence in a weighted $C^{1}$ norm. In [14] the authors consider numerical approximations to the scaled first derivative of the solution of the singularly perturbed 
two parameter problem. The method of proof is based on the argument given in [3] for the special case of $\mu=1$. However, many of the main results (e.g. [14, Lemma 5]) are stated without proof and certain crucial steps in the supplied proofs do not hold up to scrutiny (e.g. see the bound (16) in [14, Lemma 10] and note that in the left layer region [14, Lemma 7$]$ simply yields that the error is bounded by $C N^{-1}$ ). In this paper, we use a different method of proof from [3] and all the relevant details for the proofs are supplied.

In the broad context of singularly perturbed problems, there are two main classes of problems (reaction-diffusion and convection-diffusion) studied in the literature. One attraction of considering the two-parameter-problem is that this problem class encompasses both of these classes. Nevertheless, in the proofs of the main results given below, we see that this classification into two types of problem classes persists. The numerical analysis presented below re-enforces the distinction between singularly perturbed problems of reaction-diffusion type and those of convection-diffusion type.

In this paper, we measure the accuracy of the numerical approximations in an appropriately weighted $C^{1}$ norm (see the definition given in (10)). Note that not all norms are suitable for singularly perturbed problems. For example, the standard weighted energy norm

$$
\|u\|_{E}^{2}:=\varepsilon\left\|u^{\prime}\right\|_{0}^{2}+\|u\|_{0}^{2} ; \quad\|v\|_{0}^{2}:=\int_{0}^{1} v^{2}(x) d x,
$$

measures the size of the left and right layer functions $w_{L}, w_{R}$ (defined below in $(5 \mathrm{c}, 5 \mathrm{~d}))$ as small, when both parameters $\varepsilon, \mu$ are small; since

$$
\max \left\{\left\|w_{L}\right\|_{E}^{2},\left\|w_{R}\right\|_{E}^{2}\right\} \leq C \max \{\sqrt{\varepsilon}, \mu\} .
$$

However, both of these layer functions are $O(1)$ in the standard $L_{\infty}$ norm.

The paper is structured as follows. In $\S 2$, parameter-explicit a priori bounds on the derivatives of the continuous solution are established. These bounds motivate the scaling used in the definition of the scaled $C^{1}$-norm, which is the norm used to measure the error in the numerical approximations. The numerical method is constructed in $\S 3$. Section 4 is the core section, where the nodal error analysis is given. The global error analysis is conducted in $\S 5$ and numerical results for a sample problem are given in $\S 6$. Some of the proofs are deferred to the Appendices and additional technical details are available in [13].

Notation: Throughout the paper, $C$ denotes a generic constant that is independent of the singular perturbation parameters $\varepsilon, \mu$ and the number of mesh elements $N$. We adopt the following notation for the semi-norms of the solution:

$$
|z|_{D, k}:=\left\|\frac{d^{k} z}{d x^{k}}\right\|_{D}=\left\|z^{(k)}\right\|_{D}, \quad\|z\|_{D}:=\sup _{x \in D}|z(x)|, \quad D=(a, b) \subset[0,1] ;
$$

and the subscript $D$ is omitted if $D \equiv[0,1]$. The following notation appears throughout the paper:

$$
\theta:=\max \left\{1, \frac{\alpha \mu^{2}}{\gamma \varepsilon}\right\}, \quad \rho_{L}:=\max \left\{1, \frac{1}{2} \sqrt{\frac{\gamma \alpha}{\theta \varepsilon}}\right\} \quad \text { and } \quad \rho_{R}:=\max \left\{1, \sqrt{\frac{\theta \gamma \alpha}{\varepsilon}}\right\}
$$


where $\alpha$ and $\gamma$ are positive free parameters which are strictly bounded above by (1c). The analytical layer widths (defined in (9)) are denoted by $\tau_{L}, \tau_{R}$ and the computational layer widths (defined in (11b)) are denoted by $\sigma_{L}, \sigma_{R}$.

\section{Continuous problem}

Find $u \in C^{5}(\Omega) \cap C^{0}(\bar{\Omega})$ such that

$$
\begin{array}{r}
L_{\varepsilon, \mu} u:=-\varepsilon u^{\prime \prime}+\mu a(x) u^{\prime}+b(x) u=f(x), x \in \Omega:=(0,1), \\
u(0)=0, \quad u(1)=0, \\
a(x)>\alpha>0, \min _{x \in[0,1]}\left(\frac{b(x)}{a(x)}\right)>\gamma>0, \quad x \in \Omega .
\end{array}
$$

The functions $a, b$ and $f$ are assumed to be sufficiently smooth on $\Omega$ and the perturbation parameters satisfy $0<\varepsilon \leq 1,0 \leq \mu \leq 1$. Since the problem (1) is linear, there is no loss in generality in assuming zero boundary conditions. Our interest lies in the case where $\varepsilon, \mu$ are both small parameters. Given the constraint (1c), there is no loss in generality in assuming that

$$
b \pm 2 \mu \max \left\{a^{\prime}\right\}>0
$$

as the case where $\mu \geq \mu_{0}>0$, and $\mu_{0}$ is a fixed positive constant, has been dealt with in earlier publications [7].

As in [12] the problem naturally splits into the two separate cases of:

$$
0 \leq \frac{\alpha \mu^{2}}{\gamma \varepsilon} \leq 1 \quad \text { and } \quad \frac{\alpha \mu^{2}}{\gamma \varepsilon} \geq 1
$$

We refer to the first case as the reaction-dominated case and the second case as the convection-dominated case. We associate the following parameter

$$
\max \left\{1, \frac{\alpha}{\gamma \varepsilon}\right\} \geq \theta:=\max \left\{1, \frac{\alpha \mu^{2}}{\gamma \varepsilon}\right\} \geq 1 ;
$$

with this division of the parameter space $P_{\varepsilon, \mu}:=\{(\varepsilon, \mu): 0<\varepsilon \leq 1,0 \leq \mu \leq$ $1\}$. Our first result establishes preliminary parameter-explicit bounds on the continuous solution and it's derivatives.

Lemma 1. Assume $a, b, f \in C^{3}(\Omega)$, then the solution $u$ of problem (1) satisfies

$$
\begin{aligned}
\|u\| & \leq \frac{1}{\gamma \alpha}\|f\| ; \\
\sqrt{\varepsilon \theta}|u|_{1} & \leq C(1+\theta)\|u\|+C\|f\| ;
\end{aligned}
$$

and, for all $k$ such that $2 \leq k \leq 5$;

$$
\varepsilon^{k / 2}|u|_{k} \leq C \theta^{(k / 2-1)}(1+\theta)\|u\|+C \sum_{j=0}^{k-2} \varepsilon^{j / 2} \theta^{(k-j-2) / 2}|f|_{j} .
$$


Proof. We follow the argument in [11, Lemma 2.2]. By the maximum principle $\|u\| \leq C$. Given any $x \in(0,1)$, we construct an open neighbourhood $N_{x}:=$ $(p, p+r)$ such that $x \in N_{x} \subset(0,1)$. By the Mean Value Theorem, there exists a $y \in N_{x}$ such that

$$
\left|u^{\prime}(y)\right|=\left|\frac{u(p+r)-u(p)}{r}\right| \leq \frac{2\|u\|}{r} .
$$

Note that

$$
\begin{aligned}
u^{\prime}(x) & =u^{\prime}(y)+\int_{t=y}^{x} u^{\prime \prime} d t=u^{\prime}(y)+\frac{1}{\varepsilon} \int_{t=y}^{x} \mu a u^{\prime}+b u-f d t \\
& =u^{\prime}(y)+\frac{\mu}{\varepsilon}((a u)(x)-(a u)(y))-\frac{1}{\varepsilon} \int_{t=y}^{x} \mu a^{\prime} u-b u+f d t .
\end{aligned}
$$

Thus

$$
\left|u^{\prime}(x)\right| \leq C\left(\frac{1}{r}+\frac{\mu}{\varepsilon}+\frac{r}{\varepsilon}\right)\|u\|+\frac{r}{\varepsilon}\|f\| .
$$

By taking the radius $r$ of the neighbourhood $N_{x}$ to be

$$
r=\sqrt{\frac{\varepsilon \gamma}{2 \theta \alpha}}
$$

we obtain the desired bound on $|u|_{1}$. Use the differential equation (1a) to obtain the bound on $|u|_{2}$ and by differentiating both sides of the differential equation we obtain bounds on all the higher derivatives. See [13] for details.

In order to obtain parameter-uniform error estimates on the numerical approximations, constructed in later sections, we decompose the solution into regular and singular components. The regular component is constructed so that the first three derivatives of this component are bounded independently of the parameters $\varepsilon, \mu$. The solution of (1) is decomposed into the following sum

$$
u(x)=v(x)+((u-v)(0)) w_{L}(x)+\left(\left(u-v-w_{L}\right)(1)\right) w_{R}(x) ;
$$

where $w_{L}$ and $w_{R}$ satisfy homogeneous differential equations and

$$
\begin{aligned}
L_{\varepsilon, \mu} v= & f,(v(0) \text { and } v(1) \text { chosen appropriately }) \\
L_{\varepsilon, \mu} w_{R}= & 0, w_{R}(0)=0, w_{R}(1)=1 \\
L_{\varepsilon, \mu} w_{L}= & 0, w_{L}(0)=1 \\
& \text { if } \mu^{2} \leq \frac{\gamma \varepsilon}{\alpha}, w_{L}(1)=0 \text { else } w_{L}(1) \text { is chosen appropriately. }
\end{aligned}
$$

We introduce the following notation for the reduced differential operators $L_{0}, L_{\mu}$,

$$
L_{0} z:=b z \quad \text { and } \quad L_{\mu} z:=\mu a z^{\prime}+b z .
$$

In the next Theorem, we refine the bounds on the continuous solution $u$ given in Lemma 1. These sharper bounds identify both the location and the 
scale of the layers, which are used in the construction of the piecewise-uniform Shishkin mesh [3]. In addition, these bounds identify the appropriate scaling to use when estimating the error in approximating the first derivatives of the continuous solution $u$. For example, from these bounds we see that

$$
\left|u^{\prime}(x)\right| \leq C, \quad \text { for } \quad 2 \frac{\sqrt{\varepsilon \theta}}{\sqrt{\gamma \alpha}} \ln \frac{1}{\sqrt{\varepsilon \theta}} \leq x \leq 1-\sqrt{\frac{\varepsilon}{\gamma \alpha \theta}} \ln \sqrt{\frac{\theta}{\varepsilon}} .
$$

Theorem 1. Assume that $a \in C^{7}(\Omega), b, f \in C^{9}(\Omega)$. Boundary conditions $v(0), v(1)$ for the regular component $v$ can be chosen so that the derivatives of the regular component (defined in (5b)) satisfy the bounds

$$
\text { (i) } \quad|v|_{k} \leq C\left(1+\left(\sqrt{\frac{\varepsilon}{\theta}}\right)^{3-k}\right), \quad \text { for } \quad 0 \leq k \leq 5 .
$$

When the solution $u$ of problem (1) is decomposed as in (5a), the singular components $w_{L}$ and $w_{R}$ (defined in $(5 c, 5 d)$ ) satisfy the following bounds

$$
\begin{aligned}
& \left|w_{L}(x)\right| \leq C e^{-\frac{\sqrt{\gamma \alpha}}{2 \sqrt{\varepsilon \theta}} x},\left|w_{R}(x)\right| \leq C e^{-\frac{\sqrt{\gamma \alpha \theta}}{\sqrt{\varepsilon}}(1-x)} \\
& \left|\frac{d^{k} w_{R}}{d x^{k}}(x)\right| \leq C\left(\sqrt{\frac{\theta}{\varepsilon}}\right)^{k} e^{-\frac{\sqrt{\gamma \alpha \theta}}{\sqrt{\varepsilon}}(1-x)}, \quad 1 \leq k \leq 5 \\
& \left|\frac{d^{k} w_{L}}{d x^{k}}(x)\right| \leq C\left(\frac{1}{\sqrt{\varepsilon \theta}}\right)^{k}\left(1+\theta^{k-3}\right) e^{-\frac{\sqrt{\gamma \alpha}}{2 \sqrt{\varepsilon \theta}}}, \quad 1 \leq k \leq 5 .
\end{aligned}
$$

Proof. The argument follows [4, §3] closely. See Appendix A for details.

Based on the bounds (7b) and (7c), we identify the decay rates in each of the layer regions by

$$
\rho_{L}:=\max \left\{1, \frac{1}{2} \sqrt{\frac{\gamma \alpha}{\theta \varepsilon}}\right\} \quad \text { and } \quad \rho_{R}:=\max \left\{1, \sqrt{\frac{\theta \gamma \alpha}{\varepsilon}}\right\}
$$

and the associated layer widths (for the continuous solution) to be

$$
\frac{1}{\rho_{L}} \ln \rho_{L} \quad \text { and } \quad \frac{1}{\rho_{R}} \ln \rho_{R} .
$$

Note that in the reaction-dominated case (where $\theta=1$ ) the layer widths are $O(\sqrt{\varepsilon})$ at both ends and in the convection-dominated case (where $\theta>1$ ) the layer widths are $O(\mu)$ on the left and $O\left(\varepsilon \mu^{-1}\right)$ on the right.

Throughout the paper we shall assume that the parameters $\varepsilon$ and $\mu$ are such that $\rho_{L}>1$ and $\rho_{R}>1$. When $\rho_{L}=1$ then $\theta \varepsilon \geq C$, which combined with the bounds in $(7 \mathrm{c})$ indicate that there is no layer on the left (or on the right if $\left.\rho_{R}=1\right)$. This case can be analysed using classical arguments.

Note that

$$
\left|w_{L}^{\prime}(x)\right| \leq C, x \geq \frac{1}{\rho_{L}} \ln \rho_{L} \quad \text { and } \quad\left|w_{R}^{\prime}(x)\right| \leq C, x \leq 1-\frac{1}{\rho_{R}} \ln \rho_{R} .
$$


In order to establish the main parameter-uniform error bound, we define the following (slightly wider) analytical layer widths to be

$$
\tau_{L}:=\min \left\{\frac{1}{4}, \frac{2}{\rho_{L}} \ln \rho_{L}\right\} \quad, \quad \tau_{R}:=\min \left\{\frac{1}{4}, \frac{2}{\rho_{R}} \ln \rho_{R}\right\}
$$

and we choose to measure the accuracy of our numerical approximations in the following weighted $C^{1}$ norm

$$
\|v\|_{1, \chi}:=\left\|\chi v^{\prime}\right\|+\|v\|, \quad \text { where } \quad \chi(x):=\left\{\begin{array}{l}
\sqrt{\varepsilon \theta}, \text { if } \quad x \leq \tau_{L}, \\
1, \quad \text { if } \tau_{L}<x<1-\tau_{R}, \\
\sqrt{\frac{\varepsilon}{\theta}}, \text { if } x \geq 1-\tau_{R} .
\end{array}\right.
$$

\section{Discrete Problem}

A piecewise-uniform Shishkin mesh [3] is constructed as follows. The domain is subdivided into three subintervals:

$$
\left[0, \sigma_{L}\right] \cup\left[\sigma_{L}, 1-\sigma_{R}\right] \cup\left[1-\sigma_{R}, 1\right] ;
$$

where the transition parameters between the subintervals are taken to be

$$
\sigma_{L}:=\min \left\{\frac{1}{4}, \frac{2}{\rho_{L}} \ln N\right\}, \quad \sigma_{R}:=\min \left\{\frac{1}{4}, \frac{4}{\rho_{R}} \ln N\right\} .
$$

Throughout most of the analysis in this paper we shall deal with the case where

$$
\sigma_{R} \leq \sigma_{L}<1 / 4
$$

On each of the two end subintervals a uniform mesh with $\frac{N}{4}$ mesh-intervals is placed. The remainder of the mesh points are placed in the inner coarse mesh region. Throughout the paper, the mesh steps are denoted by

$$
h_{i}:=x_{i}-x_{i-1}, \quad 2 \bar{h}_{i}:=x_{i+1}-x_{i-1}
$$

and $h_{L}, H, h_{R}$ denote the mesh width in the left fine mesh, the central coarse mesh and the right fine mesh, respectively. The subsequent layer-adapted piecewise uniform mesh will be denoted by $\omega_{\varepsilon, \mu}^{N}$. By this choice of transition parameters, we see that for $k=1,2$,

$$
\begin{aligned}
h_{L}^{k}\left|\frac{d^{k} w_{L}}{d x^{k}}(x)\right| & \leq C\left(N^{-1} \ln N\right)^{k}, \quad x<\sigma_{L} ; \\
h_{R}^{k}\left|\frac{d^{k} w_{R}}{d x^{k}}(x)\right| & \leq C\left(N^{-1} \ln N\right)^{k}, \quad x>1-\sigma_{R} .
\end{aligned}
$$

The discrete problem is of the form:

$$
\begin{aligned}
L^{N} U\left(x_{i}\right):= & \left(-\varepsilon \delta^{2}+\mu a D^{-}+b\right) U\left(x_{i}\right)=f\left(x_{i}\right), \quad x_{i} \in \omega_{\varepsilon, \mu}^{N} \\
& U(0)=u(0), \quad U(1)=u(1)
\end{aligned}
$$


where $D^{-}$denotes the backward difference operator and $\delta^{2}$ is the standard replacement to the second derivative on a non-uniform mesh. ${ }^{1}$ Note that this finite difference operator $L^{N}$ satisfies a discrete minimum principle: If $Z$ is a mesh function such that $Z(0) \geq 0, Z(1) \geq 0$ and $L^{N} Z\left(x_{i}\right) \geq 0, x_{i} \in \omega_{\varepsilon, \mu}^{N}$, then $Z\left(x_{i}\right) \geq 0, x_{i} \in \bar{\omega}_{\varepsilon, \mu}^{N}$.

Analogous to the continuous solution, the discrete solution can be decomposed into the sum $U=V+W_{L}+W_{R}$, where the components are the solutions of the problems

$$
\begin{aligned}
\left(L^{N} V\right)\left(x_{i}\right) & =f\left(x_{i}\right), \quad V(0)=v(0), \quad V(1)=v(1) \\
\left(L^{N} W_{L}\right)\left(x_{i}\right) & =0, \quad W_{L}(0)=w_{L}(0), \quad W_{L}(1)=w_{L}(1) \\
\left(L^{N} W_{R}\right)\left(x_{i}\right) & =0, \quad W_{R}(0)=w_{R}(0)=0, \quad W_{R}(1)=w_{R}(1)
\end{aligned}
$$

In the next result, we establish bounds on the discrete layer components, which are the discrete counterparts to the bounds (7a) established on the continuous layer components. Note the different decay rates in $(15 \mathrm{a}, 15 \mathrm{~b})$, which arise from the fact that the discrete second derivatives of the barrier functions $\Psi_{L}, \Psi_{R}$ are positive and have the different scales $\varepsilon \delta^{2} \Psi_{L}=O(1)$ and $\varepsilon \delta^{2} \Psi_{R}=O(\theta)$.

Theorem 2. Assume (12). We have the following bounds on $W_{L}$ and $W_{R}$

$$
\begin{aligned}
& \left|W_{L}\left(x_{j}\right)\right| \leq C \prod_{i=1}^{j}\left(1+\rho_{L} h_{i}\right)^{-1}=: \Psi_{L}\left(x_{j}\right), \quad \Psi_{L}(0):=C \\
& \left|W_{R}\left(x_{j}\right)\right| \leq C \prod_{i=j+1}^{N}\left(1+0.5 \rho_{R} h_{i}\right)^{-1}=: \Psi_{R}\left(x_{j}\right), \quad \Psi_{R}(1):=C .
\end{aligned}
$$

Proof. (i) We begin with the left boundary layer function $W_{L}$. Recall that $w_{L}(1) \neq 0$, when $\theta \neq 1$. In this special case, observe that

$$
\rho_{L}=\frac{\gamma}{2 \mu}, \quad \text { if } \theta \neq 1
$$

From this and the inequality $e^{-x} \leq(1+x)^{-1}, x>0$, one can deduce that $\Psi_{L}(1) \geq C e^{-\frac{\gamma}{2 \mu}}$, when $\theta \neq 1$. Hence for all $\theta, \Psi_{L}(1) \geq\left|w_{L}(1)\right|$ and $\Psi_{L}(0) \geq$ $\left|w_{L}(0)\right|$. Next we consider the interior mesh points.

Consider $\Phi_{L}^{ \pm}\left(x_{j}\right):=\Psi_{L}\left(x_{j}\right) \pm W_{L}\left(x_{j}\right)$, where $\Psi_{L}\left(x_{j}\right)$ is defined in (15a). Using the properties $\Psi_{L}\left(x_{j}\right)>0$,

$$
D^{-} \Psi_{L}\left(x_{j}\right)=-\rho_{L} \Psi_{L}\left(x_{j}\right)<0, \quad \text { and } \quad \delta^{2} \Psi_{L}\left(x_{j}\right)=\rho_{L}^{2} \Psi_{L}\left(x_{j+1}\right) \frac{h_{j+1}}{\bar{h}_{j}}>0,
$$

\footnotetext{
${ }^{1}$ The finite difference operators $D^{+}, D^{-}, \delta^{2}$ are, respectively, defined by $D^{+} Z\left(x_{i}\right):=\frac{Z\left(x_{i+1}\right)-Z\left(x_{i}\right)}{h_{i+1}} ; D^{-} Z\left(x_{i}\right):=D^{+} Z\left(x_{i-1}\right) ; \delta^{2} Z\left(x_{i}\right):=\frac{D^{+} Z\left(x_{i}\right)-D^{-} Z\left(x_{i}\right)}{\bar{h}_{i}}$.
} 
we obtain

$$
\begin{gathered}
L^{N} \Phi_{L}^{ \pm}\left(x_{j}\right) \geq \\
\Psi_{L}\left(x_{j+1}\right)\left(2 \varepsilon \rho_{L}^{2}\left(1-\frac{h_{j+1}}{2 h_{j}}\right)+\left(b-2 \varepsilon \rho_{L}^{2}-\mu a \rho_{L}+\left(b-\mu a \rho_{L}\right) \rho_{L} h_{j+1}\right) .\right.
\end{gathered}
$$

Note that $\left(b-\mu a \rho_{L}\right)=a\left(\frac{b}{a}-\mu \rho_{L}\right) \geq a \gamma 0.5$ and

$$
b-2 \varepsilon \rho_{L}^{2}-\mu a \rho_{L}=a\left(\frac{b}{a}-\frac{\gamma \alpha}{2 \theta a}-\frac{\mu}{2} \sqrt{\frac{\gamma \alpha}{\theta \varepsilon}}\right) \geq a \gamma\left(1-\frac{1}{2 \theta}-\frac{1}{2} \sqrt{\frac{\alpha \mu^{2}}{\gamma \theta \varepsilon}}\right) \geq 0 .
$$

Now using the discrete minimum principle we obtain the required bound (15a).

(ii) The same argument is applied to bound $W_{R}$. Using

$$
D^{+} \Psi_{R}\left(x_{j}\right)=0.5 \rho_{R} \Psi_{R}\left(x_{j}\right) \quad \text { and } \quad \delta^{2} \Psi_{R}\left(x_{j}\right)=\frac{\rho_{R}^{2}}{4\left(1+0.5 \rho_{R} h_{j}\right)} \Psi_{R}\left(x_{j}\right) \frac{h_{j}}{\overline{h_{j}}}
$$

we obtain (noting $a \mu-\varepsilon \rho_{R} \geq 0$ ) the inequality

$$
L^{N} \Psi_{R}\left(x_{j}\right) \geq \Psi_{R}\left(x_{j-1}\right)\left(-0.5 \varepsilon \rho_{R}^{2}+\left(b+0.5 \mu a \rho_{R}\right)\left(1+0.5 \rho_{R} h_{j}\right)\right) \geq 0 .
$$

Use the discrete minimum principle to obtain the required bound (15b).

From these bounds we deduce that, for all $x_{i} \leq 1-\sigma_{R}$,

$$
\left|W_{R}\left(x_{i}\right)\right| \leq\left|W_{R}\left(1-\sigma_{R}\right)\right| \leq C N^{-2} ;
$$

and, at the left end, for all $x_{i} \geq \sigma_{L}$

$$
\left|W_{L}\left(x_{i}\right)\right| \leq\left|W_{L}\left(\sigma_{L}\right)\right| \leq C N^{-2} .
$$

Hence, outside their corresponding layer regions, the discrete layer functions $W_{L}, W_{R}$ are small, from a computational perspective.

\section{Nodal error analysis}

We denote the nodal error and associated truncation error, respectively, by

$$
e\left(x_{i}\right):=U\left(x_{i}\right)-u\left(x_{i}\right), \quad \text { and } \mathcal{T}\left(x_{i}\right):=L^{N} e\left(x_{i}\right) .
$$

When bounding the local truncation error, we utilize the following standard bounds at all mesh points, excluding the transition points: For all $x_{i} \neq \sigma_{L}, 1-\sigma_{R}$

$$
\begin{aligned}
& \left.\mid L^{N}(U-u)\left(x_{i}\right)\right) \mid \leq \\
& C h_{i}\left(\varepsilon \max \left\{\left\|u^{(3)}\right\|_{\left[x_{i-1}, x_{i+1}\right]}, h_{i}\left\|u^{(4)}\right\|_{\left[x_{i-1}, x_{i+1}\right]}\right\}+\mu\left\|u^{(2)}\right\|_{\left[x_{i-1}, x_{i}\right]}\right),
\end{aligned}
$$

and at all mesh points

$$
\left.\mid L^{N}(U-u)\left(x_{i}\right)\right) \mid \leq C \varepsilon\left(h_{i}+h_{i+1}\right)\left\|u^{(3)}\right\|_{\left[x_{i-1}, x_{i+1}\right]}+C \mu h_{i}\left\|u^{(2)}\right\|_{\left[x_{i-1}, x_{i}\right]} .
$$


We define the discrete error flux to be

$$
\mathcal{U}_{i}:=D^{-} e\left(x_{i}\right), \quad \text { if } 0<x_{i} \leq 1 .
$$

On a piecewise-uniform mesh the finite difference operators $\delta^{2}$ and $D^{-}$do not commute on a non-uniform mesh. Based on this observation, we define a new finite difference operator $\hat{\delta}^{2}$ by

$$
\hat{\delta}^{2} Z_{i}:=\frac{1}{\hbar_{i}}\left(\frac{h_{i+1}}{h_{i}} D^{+}-\frac{\hbar_{i}}{\hbar_{i-1}} D^{-}\right) Z_{i},
$$

which has the property that

$$
\hat{\delta}^{2} D^{-} Z_{i} \equiv D^{-} \delta^{2} Z_{i}
$$

on an arbitrary mesh. Note that the second order operator is $\hat{\delta}^{2}$ on the left and $\delta^{2}$ on the right of this identity. Hence, this identity is not a statement of commutativity. Note the following identity (Discrete derivatives of a product of two mesh functions)

$$
D^{-}\left(P_{i} Q_{i}\right) \equiv P_{i} D^{-} Q_{i}+Q_{i-1} D^{-} P_{i} .
$$

Using these identities and $D^{-}\left(L^{N} e\left(x_{i}\right)\right)=D^{-} \mathcal{T}\left(x_{i}\right)$, we see that for all mesh points within the region $\left(h_{1}, 1\right)$, the discrete flux $\mathcal{U}_{i}$ satisfies

$$
\hat{L}^{N} \mathcal{U}\left(x_{i}\right)=D^{-} \mathcal{T}\left(x_{i}\right)-e\left(x_{i-1}\right) D^{-} b\left(x_{i}\right), \quad x_{i} \in\left(h_{1}, 1\right) ;
$$

where for the internal mesh points

$$
\hat{L}^{N} Z\left(x_{i}\right):=\left(-\varepsilon \hat{\delta}^{2}+\mu a\left(x_{i-1}\right) D^{-}+\left(b+\mu D^{-} a\right)\left(x_{i}\right) I\right) Z\left(x_{i}\right),
$$

and for the end points $\hat{L}^{N} Z\left(x_{i}\right):=Z\left(x_{i}\right)$ for $x_{i}=h_{1}, 1$.

Based on classical bounds on the truncation error, at any mesh point,

$$
\begin{aligned}
\left|D^{-}\left(u^{\prime}-D^{-} u\right)\left(x_{i}\right)\right| & \leq C\left(1+\frac{h_{i-1}}{h_{i}}\right)\left\|u^{(2)}\right\|_{\left[x_{i-2}, x_{i}\right]}, \\
\left|D^{-}\left(u^{\prime \prime}-\delta^{2} u\right)\left(x_{i}\right)\right| & \leq C\left(1+\frac{h_{i-1}+h_{i+1}}{h_{i}}\right)\left\|u^{(3)}\right\|_{\left[x_{i-2}, x_{i+1}\right]} .
\end{aligned}
$$

In addition, if $h_{i-1}=h_{i}$, then

$$
\left|D^{-}\left(u^{\prime}-D^{-} u\right)\left(x_{i}\right)\right| \leq C h_{i}\left\|u^{(3)}\right\|_{\left[x_{i-2}, x_{i}\right]},
$$

and if $h_{i-1}=h_{i}=h_{i+1}$, then

$$
\left|D^{-}\left(u^{\prime \prime}-\delta^{2} u\right)\left(x_{i}\right)\right| \leq C \max \left\{h_{i}\left\|u^{(4)}\right\|_{\left[x_{i-1}, x_{i+1}\right]}, h_{i}^{2}\left\|u^{(5)}\right\|_{\left[x_{i-2}, x_{i+1}\right]}\right\} .
$$

Based on the assumption (2) the discrete operator $\hat{L}^{N}$ (22) satisfies a discrete comparison principle. To bound the error in the discrete flux $\mathcal{U}_{i}$, we employ a standard stability and consistency argument using the operator $\hat{L}^{N}$ (and not 
the operator $\left.L^{N}\right)$. To this end we bound $D^{-}\left(L^{N}\left(e\left(x_{i}\right)\right)\right)$ and the error fluxes at the endpoints of the interval $\left(h_{1}, 1\right)$. The main complication in the analysis is the construction of suitable discrete barrier functions.

Now we deduce bounds on the regular $\mathcal{V}:=D^{-}(V-v)$ and the singular components $\mathcal{W}_{L}:=D^{-}\left(W_{L}-w_{L}\right), \mathcal{W}_{R}:=D^{-}\left(W_{R}-w_{R}\right)$ of the discrete error flux $\mathcal{U}$. We begin with the singular component $W_{L}$, as in this case the analysis is a little easier. We will need an appropriate bound on the boundary error flux $\left|D^{+}\left(W_{L}-w_{L}\right)(0)\right|$. We achieve this by sharping the standard nodal error bound $\left|\left(W_{L}-w_{L}\right)\left(x_{i}\right)\right| \leq C N^{-1} \ln N$, within the layer region on the left, to reflect the fact that $\left(W_{L}-w_{l}\right)(0)=0$.

Lemma 2. Assume (12). For sufficiently large $N$,

$$
\sqrt{\varepsilon \theta}\left|D^{+}\left(W_{L}-w_{L}\right)(0)\right| \leq C N^{-1}(\ln N),
$$

where $W_{L}$ is the solution of $(14 b)$ and $w_{L}$ is the solution of $(5 d)$.

Proof. (i) In the convection-diffusion case of $\theta>1$, we introduce the following linear discrete barrier function

$$
B\left(x_{i}\right):=C \frac{x_{i}}{\mu}\left\|L^{N}\left(W_{L}-w_{L}\right)\right\|_{\left(0, \sigma_{L}\right)}+C N^{-2},
$$

so that $L^{N} B \geq C\left\|L^{N}\left(W_{L}-w_{L}\right)\right\|$. Note that this barrier function cannot be used in the reaction-diffusion case when $\theta=1$, as it involves the multiple $\mu^{-1}$. Here $\left\|L^{N}\left(W_{L}-w_{L}\right)\right\|_{\left(0, \sigma_{L}\right)}$ is the truncation error associated with the left singular component $w_{L}$. In the boundary layer region $\left(0, \sigma_{L}\right)$, using $(7 \mathrm{c})$ and the standard truncation error bounds (17) we have that

$$
\left\|L^{N}\left(W_{L}-w_{L}\right)\right\|_{\left(0, \sigma_{L}\right)} \leq C\left(\frac{1}{\theta}+\frac{\mu}{\sqrt{\varepsilon \theta}}\right) N^{-1} \ln N .
$$

In addition, by (7a) and (16b) we can deduce that $\left(W_{L}-w_{L}\right)(0)=0$ and $\left|\left(W_{L}-w_{L}\right)\left(\sigma_{L}\right)\right| \leq C N^{-2}$. From the discrete minimum principle, we have that

$\left|\left(W_{L}-w_{L}\right)\left(x_{i}\right)\right| \leq B\left(x_{i}\right) \leq C x_{i}\left(\frac{1}{\mu \theta}+\frac{1}{\sqrt{\varepsilon \theta}}\right) N^{-1} \ln N+C N^{-2}, \quad$ for $x_{i} \in\left[0, \sigma_{L}\right]$,

and, in particular,

$$
\left|\left(W_{L}-w_{L}\right)\left(h_{L}\right)\right| \leq C h_{L}\left(\frac{1}{\mu \theta}+\frac{1}{\sqrt{\varepsilon \theta}}\right) N^{-1} \ln N+C N^{-2} .
$$

Therefore, when $\theta>1$,

$$
\begin{aligned}
\sqrt{\varepsilon \theta}\left|D^{+}\left(W_{L}-w_{L}\right)(0)\right| & \leq C \sqrt{\varepsilon \theta}\left(\frac{1}{\theta \mu}+\frac{1}{\sqrt{\varepsilon \theta}}\right) N^{-1} \ln N+C N^{-1} \\
& \leq C N^{-1}(\ln N) .
\end{aligned}
$$


(ii) In the reaction-diffusion case, where $\theta=1$, we utilize the bound (16b) to allow us confine the truncation error estimate (17) to the fine uniform mesh. For all mesh points $x_{i} \in\left(0, \sigma_{L}\right)$, this yields

$$
\left|L^{N}\left(W_{L}-w_{L}\right)\left(x_{i}\right)\right| \leq C\left(N^{-1} \ln N+\frac{\mu}{\sqrt{\varepsilon}}\right)\left(N^{-1} \ln N\right) .
$$

Consider the following discrete barrier function

$$
C\left(\frac{N^{-1} \ln N}{\beta}+\frac{x_{i}}{\sqrt{\varepsilon}}\right)\left(N^{-1} \ln N\right)+C N^{-2}
$$

and using the discrete minimum principle we get that

$$
\left|\left(W_{L}-w_{L}\right)\left(h_{L}\right)\right| \leq C\left(\frac{\left(N^{-1} \ln N\right)^{2}}{\beta}+\left(N^{-1} \ln N\right) \frac{h_{L}}{\sqrt{\varepsilon}}\right)+C N^{-2} .
$$

Now we have, for the case when $\theta=1$,

$$
\begin{aligned}
\sqrt{\varepsilon}\left|D^{+}\left(W_{L}-w_{L}\right)(0)\right| & \leq C \frac{\sqrt{\varepsilon}}{h_{L}}\left(\frac{\left(N^{-1} \ln N\right)^{2}}{\beta}+\left(N^{-1} \ln N\right) \frac{h_{L}}{\sqrt{\varepsilon}}\right)+C N^{-1} \\
& \leq C N^{-1} \ln N
\end{aligned}
$$

Hence we have completed the proof for both $\theta>1$ and $\theta=1$.

Note that by examining the bounds in the above Lemma, we have the nodal error bound

$$
\left|\left(W_{L}-w_{L}\right)\left(x_{i}\right)\right| \leq C N^{-1}(\ln N)^{2} .
$$

Theorem 3. Assume (12). We have the bounds

$$
\begin{aligned}
\sqrt{\varepsilon \theta}\left|D^{-}\left(W_{L}-w_{L}\right)\left(x_{i}\right)\right| & \leq C N^{-1} \ln N, \quad \text { if } 0<x_{i} \leq \sigma_{L} \\
\left|D^{-}\left(W_{L}-w_{L}\right)\left(x_{i}\right)\right| & \leq C N^{-1}, \quad \text { if } \quad \sigma_{L}<x_{i} \leq 1-\sigma_{R} \\
\sqrt{\frac{\varepsilon}{\theta}}\left|D^{-}\left(W_{L}-w_{L}\right)\left(x_{i}\right)\right| & \leq C N^{-1}, \quad \text { if } 1-\sigma_{R}<x_{i} \leq 1
\end{aligned}
$$

where $W_{L}$ is the solution of (14b) and $w_{L}$ is the solution of (5d).

Proof. Using the bounds (7a) and (16b), respectively, on $w_{L}$ and $W_{L}$ we see that outside the left layer region

$$
\left|\left(W_{L}-w_{L}\right)\left(x_{i}\right)\right| \leq C N^{-2}, \quad x_{i} \geq \sigma_{L} .
$$

Combining this bound with the fact that $h_{R}=C \sqrt{\frac{\varepsilon}{\theta}} N^{-1} \ln N$ we deduce that

$$
\begin{aligned}
\left|D^{-}\left(W_{L}-w_{L}\right)\left(x_{i}\right)\right| & \leq C N^{-1} \quad \text { if } \quad \sigma_{L}<x_{i} \leq 1-\sigma_{R} \\
\sqrt{\frac{\varepsilon}{\theta}}\left|D^{-}\left(W_{L}-w_{L}\right)\left(x_{i}\right)\right| & \leq C N^{-1} \quad \text { if } \quad x_{i}>1-\sigma_{R} .
\end{aligned}
$$


It remains to establish the bound in the left layer region, where the derivatives of the left boundary layer function $w_{L}$ are significant. From (7a) we have that

$$
\left|w_{L}\left(\sigma_{L}-h_{L}\right)\right| \leq C e^{\frac{\sqrt{\gamma \alpha} h_{L}}{2 \sqrt{\varepsilon \theta}}} e^{\frac{-\sqrt{\gamma \alpha} \sigma}{2 \sqrt{\varepsilon \theta}}} \leq C N^{-2} ;
$$

and using Theorem 2, with $\rho_{L}:=\sqrt{\frac{\gamma \alpha}{4 \theta \varepsilon}}$ it follows that

$$
\left|W_{L}\left(\sigma_{L}-h_{L}\right)\right| \leq C\left(1+\rho_{L} h_{L}\right)\left(1+\rho_{L} h_{L}\right)^{-\frac{N}{4}} \leq C N^{-2} .
$$

Repeat the earlier argument to get that

$$
\sqrt{\varepsilon \theta}\left|D^{-}\left(W_{L}-w_{L}\right)\left(\sigma_{L}\right)\right| \leq C \frac{\sqrt{\theta \varepsilon}}{h_{L}} N^{-2} \leq C N^{-1} .
$$

Using the truncation error bounds $(23)$ in the region $\left(0, \sigma_{L}\right)$ we have

$$
\left|\hat{L}^{N} D^{-}\left(W_{L}-w_{L}\right)\left(x_{i}\right)\right| \leq \frac{C}{\sqrt{\varepsilon \theta}} N^{-1} \ln N .
$$

Complete the proof using the discrete constant barrier function $\frac{N^{-1} \ln N}{\sqrt{\varepsilon \theta}}$, Lemma 2 , the lower bound $b>\gamma \alpha$ and the end-point bound of

$$
\sqrt{\varepsilon \theta}\left|D^{-}\left(W_{L}-w_{L}\right)\left(\sigma_{L}\right)\right| \leq C N^{-1} .
$$

The analysis is more elaborate in the case of the right layer component. We first need an appropriate bound on the outgoing error flux $\left|D^{-}\left(W_{R}-w_{R}\right)(1)\right|$. We again achieve this by sharping the standard nodal error bound $\mid\left(W_{R}-\right.$ $\left.w_{R}\right)\left(x_{i}\right) \mid \leq C N^{-1} \ln N$, within the layer region on the right, to reflect the fact that $\left(W_{R}-w_{R}\right)(1)=0$.

Lemma 3. Assume (12). For sufficiently large N,

$$
\sqrt{\frac{\varepsilon}{\theta}}\left|D^{-}\left(W_{R}-w_{R}\right)(1)\right| \leq C N^{-1}(\ln N)^{2},
$$

where $W_{R}$ is the solution of (14c) and $w_{R}$ is the solution of (5c).

Proof. Consider the discrete function $\psi\left(x_{i}\right)$ defined by

$$
\begin{aligned}
& -\varepsilon \delta^{2} \psi+\sqrt{\varepsilon \theta} A D^{-} \psi=0, x_{i} \in\left(1-\sigma_{R}, 1\right), \\
& \psi\left(1-\sigma_{R}\right)=1, \psi(1)=0 ; \quad A \geq\|a\| \sqrt{\frac{\gamma}{\alpha}} .
\end{aligned}
$$

Observe that

$$
\psi\left(x_{i}\right)=\frac{1-(1+\rho)^{i-N}}{1-(1+\rho)^{-N / 4}}, \quad \text { where } \rho:=\sqrt{\frac{\theta}{\varepsilon}} A h_{R} .
$$


Note also that

$$
D^{-} \psi\left(x_{i}\right)<0 \quad \text { and } \quad(1+\rho)^{-N / 4} \leq\left(1+\frac{4 \ln N}{N}\right)^{-N / 4} \leq C N^{-1} .
$$

Hence $\psi\left(x_{i}\right) \leq C\left(1-(1+\rho)^{i-N}\right)$ for $N$ sufficiently large. Now we define a barrier function to deduce appropriate bounds for $\mathcal{W}_{N}$. First, we note that

$$
L^{N}\left(x_{i}-1+\sigma_{R} \psi\left(x_{i}\right)\right) \geq \mu a\left(x_{i}\right)+\sigma_{R}\left(\mu a\left(x_{i}\right)-\sqrt{\varepsilon \theta} A\right) D^{-} \psi\left(x_{i}\right) \geq \mu \alpha .
$$

(i) When $\theta>1$, define the following discrete barrier function

$$
B\left(x_{i}\right):=C \mu^{-1}\left\|L^{N}\left(W_{R}-w_{R}\right)\right\|_{\left(1-\sigma_{R}\right)}\left(x_{i}-1+\sigma_{R} \psi\left(x_{i}\right)\right)+C N^{-2},
$$

where $L^{N}\left(W_{R}-w_{R}\right)$ is the truncation error associated with the singular component $w_{R}$. In the boundary layer region

$$
\left\|L^{N}\left(W_{R}-w_{R}\right)\right\|_{\left(1-\sigma_{R}, 1\right)} \leq C\left(\theta+\mu \sqrt{\frac{\theta}{\varepsilon}}\right) N^{-1} \ln N .
$$

Using the discrete maximum principle we then have that, for $x_{i} \in\left[1-\sigma_{R}, 1\right]$

$$
\left|\left(W_{R}-w_{R}\right)\left(x_{i}\right)\right| \leq B\left(x_{i}\right) \leq C\left(\frac{\theta}{\mu}+\sqrt{\frac{\theta}{\varepsilon}}\right) N^{-1} \ln N\left(x_{i}-1+\sigma_{R} \psi\left(x_{i}\right)\right)+C N^{-2}
$$

and

$$
\left|\left(W_{R}-w_{R}\right)\left(1-h_{R}\right)\right| \leq C h_{R}\left(\frac{\theta}{\mu}+\sqrt{\frac{\theta}{\varepsilon}}\right) N^{-1}(\ln N)^{2}+C N^{-2} .
$$

Therefore, when $\theta>1$,

$$
\sqrt{\frac{\varepsilon}{\theta}}\left|\mathcal{W}_{N}\right| \leq C \frac{\sqrt{\varepsilon}}{\sqrt{\theta}}\left(\frac{\theta}{\mu}+\sqrt{\frac{\theta}{\varepsilon}}\right) N^{-1}(\ln N)^{2}+C N^{-1} \leq C N^{-1}(\ln N)^{2} .
$$

(ii) In the other case, where $\theta=1$, we can use the truncation error bound (17) in the boundary layer region $\left(1-\sigma_{R}, 1\right)$,

$$
\left|L^{N}\left(W_{R}-w_{R}\right)\left(x_{i}\right)\right| \leq C\left(N^{-1} \ln N+\frac{\mu}{\sqrt{\varepsilon}}\right) N^{-1} \ln N .
$$

Using the discrete maximum principle we can deduce that

$$
\left|\left(W_{R}-w_{R}\right)\left(x_{i}\right)\right| \leq C \frac{\left(N^{-1} \ln N\right)^{2}}{\beta}+\left(x_{i}-1+\sigma_{R} \psi\left(x_{i}\right)\right) \frac{N^{-1} \ln N}{\sqrt{\varepsilon}},
$$

which yields the required result for the case of $\theta=1$.

In passing, we note that the nodal error bound

$$
\left|\left(W_{R}-w_{R}\right)\left(x_{i}\right)\right| \leq C N^{-1}(\ln N)^{2},
$$

follows from the bounds established in the above Lemma 
Theorem 4. Assume (12). We have the bounds

$$
\begin{aligned}
\sqrt{\varepsilon \theta}\left|D^{-}\left(W_{R}-w_{R}\right)\left(x_{i}\right)\right| & \leq C N^{-1}, \quad \text { if } \quad x_{i} \leq \sigma_{L}, \\
\left|D^{-}\left(W_{R}-w_{R}\right)\left(x_{i}\right)\right| & \leq C N^{-1}, \quad \text { if } \quad \sigma_{L}<x_{i} \leq 1-\sigma_{R}, \\
\sqrt{\frac{\varepsilon}{\theta}}\left|D^{-}\left(W_{R}-w_{R}\right)\left(x_{i}\right)\right| & \leq C N^{-1}(\ln N)^{2}, \quad \text { if } \quad x_{i}>1-\sigma_{R} ;
\end{aligned}
$$

where $W_{R}$ is the solution of (14c) and $w_{R}$ is the solution of (5c).

Proof. Using the bounds (7a) and (16a) on $w_{R}$ and $W_{R}$, we see that outside the layer region $\left(1-\sigma_{R}, 1\right)$ we have

$$
\left|\left(W_{R}-w_{R}\right)\left(x_{i}\right)\right| \leq C N^{-2}, \quad 0 \leq x_{i} \leq 1-\sigma_{R} .
$$

Using this bound along with the mesh step $h_{L}=C \sqrt{\varepsilon \theta} N^{-1} \ln N$, we deduce that

$$
\begin{aligned}
\sqrt{\varepsilon \theta}\left|D^{-}\left(W_{R}-w_{R}\right)\left(x_{i}\right)\right| & \leq C N^{-1}, \quad \text { if } \quad x_{i} \leq \sigma_{L}, \\
\left|D^{-}\left(W_{R}-w_{R}\right)\left(x_{i}\right)\right| & \leq C N^{-1}, \quad \text { if } \quad \sigma_{L}<x_{i} \leq 1-\sigma_{R} .
\end{aligned}
$$

When $x_{i}=1-\sigma_{R}+h_{R}, 1-\sigma_{R}+2 h_{R}$ we also have

$$
\left|w_{R}\left(x_{i}\right)\right| \leq C e^{\frac{2 \sqrt{\gamma \alpha \theta} h_{R}}{\sqrt{\varepsilon}}} e^{-\frac{\sqrt{\gamma \alpha \theta \sigma_{R}}}{\sqrt{\varepsilon}}} \leq C N^{-4}
$$

and using Theorem 2, with $\rho_{R}:=\sqrt{\frac{\theta \gamma \alpha}{\varepsilon}}$ we have

$$
\left|W_{R}\left(x_{i}\right)\right| \leq C\left(1+0.5 \rho_{R} h_{R}\right)^{2}\left(1+0.5 \rho_{R} h_{R}\right)^{-\frac{N}{4}} \leq C N^{-2} .
$$

We therefore have established that

$$
\sqrt{\frac{\varepsilon}{\theta}}\left|\mathcal{W}_{\mathcal{R}}\right| \leq C N^{-1}, \quad x_{i}=1-\sigma_{R}+h_{R}, 1-\sigma_{R}+2 h_{R} .
$$

In the region $\left(1-\sigma_{R}+h_{R}, 1\right)$, using the truncation error bounds (23) and the exponential bounds in Theorem 1 we see that

$$
\begin{aligned}
\hat{L}^{N, M} \mathcal{W}_{\mathcal{R}} \leq & C \sqrt{\frac{\varepsilon}{\theta}} N^{-1} \ln N\left(\varepsilon \sqrt{\frac{\varepsilon}{\theta}}\left(\sqrt{\frac{\theta}{\varepsilon}}\right)^{5}+\mu\left(\sqrt{\frac{\theta}{\varepsilon}}\right)^{3}\right) e^{-\sqrt{\frac{\gamma \alpha \theta}{\varepsilon}}(1-x)} \\
& +C N^{-1} \ln N \\
\leq & C \sqrt{\frac{\theta}{\varepsilon}} N^{-1} \ln N\left(\theta+\sqrt{\frac{\mu^{2} \theta}{\varepsilon}}\right) e^{-\sqrt{\frac{\gamma \alpha \theta}{\varepsilon}}\left(1-x_{i+1}\right)}+C N^{-1} \ln N .
\end{aligned}
$$

In the case of $\theta=1$, this truncation error bound simplifies to

$$
\left|\hat{L}^{N} \mathcal{W}_{\mathcal{R}}\right| \leq C \frac{N^{-1} \ln N}{\sqrt{\varepsilon}}, \quad \text { if } \quad \theta=1,
$$


and the result follows using a constant discrete barrier function.

When $\theta>1$, the truncation error bound is of the form

$$
\left|\hat{L}^{N} \mathcal{W}_{\mathcal{R}}\left(x_{i}\right)\right| \leq C \sqrt{\frac{\theta}{\varepsilon}} N^{-1} \ln N \theta e^{-\sqrt{\frac{\gamma \alpha \theta}{\varepsilon}}\left(1-x_{i+1}\right)}+C N^{-1} \ln N
$$

Consider the discrete barrier function

$$
\sqrt{\frac{\theta}{\varepsilon}} N^{-1} \ln N\left(1+\left(1+h_{R} \sqrt{\frac{\gamma \alpha \theta}{\varepsilon}}\right)^{i+1-N}\right)
$$

and use the strict inequality $a(x)>\alpha$ and $(1+t)^{-1} \geq e^{-t}$ to get the required result.

We next examine the error associated with the regular component.

Lemma 4. For the discrete regular component $V$ and the continuous regular component $v$ we have the bound

$$
\left|D^{+}(V-v)(0)\right| \leq C N^{-1}, \quad \sqrt{\frac{\varepsilon}{\theta}}\left|D^{-}(V-v)(1)\right| \leq C N^{-1} .
$$

Proof. The proof is given in Appendix B.

Within the proof of Lemma 4, one can see that we have established the nodal error bound $\|V-v\| \leq C N^{-1} x_{i}+C N^{-2}$. Using the corresponding earlier bounds on the nodal error on the layer components, we now have the parameter-uniform nodal error bound

$$
\|U-u\|_{\Omega^{N}} \leq C N^{-1}(\ln N)^{2} .
$$

In the next theorem, the definition of $\tilde{\delta}^{2}$ comes into play into the numerical analysis for the first time, as the consistency bound is derived over the entire (non-uniform) mesh. The use of the operator $\tilde{\delta}^{2}$ results in isolated jumps in the truncation error at the four mesh points $\sigma_{L}, \sigma_{L}+H, 1-\sigma_{R}+h_{R}, 1-\sigma_{R}$.

Theorem 5. Assume (12). We have

$$
\begin{aligned}
\left|D^{-}(V-v)\left(x_{i}\right)\right| & \leq C N^{-1}, \quad \text { if } x_{i} \leq \sigma_{L} \\
\left|D^{-}(V-v)\left(x_{i}\right)\right| & \leq C N^{-1}(\ln N)^{2}, \text { if } 1-\sigma_{L}<x_{i} \leq 1-\sigma_{R}, \\
\sqrt{\frac{\varepsilon}{\theta}}\left|D^{-}(V-v)\left(x_{i}\right)\right| & \leq C N^{-1}(\ln N)^{2}, \text { if } x_{i}>1-\sigma_{R} .
\end{aligned}
$$

Proof. The proof is given in Appendix C.

Given the bounds in Theorems 3,4 and 5, it only remains to remove the scaling factors in certain parts of the layer regions, in the particular case where the analytical layer width is thinner than the computational layer width. That is, if $\tau_{L} \leq \sigma_{L}$ (or $\tau_{R} \leq \sigma_{R}$ ) then we need to remove the scaling factor $\sqrt{\varepsilon \theta}$ (or $\sqrt{\frac{\varepsilon}{\theta}}$ ) from the bounds within the region $\tau_{L}<x_{i}<\sigma_{L}\left(\right.$ or $\left.1-\sigma_{R}<x_{i}<1-\tau_{R}\right)$. 
Theorem 6. Assume (12). We have the scaled nodal error bounds

$$
\begin{aligned}
\sqrt{\varepsilon \theta}\left|D^{-}(U-u)\left(x_{i}\right)\right| & \leq C N^{-1}, \quad \text { if } x_{i} \leq \tau_{L}, \\
\left|D^{-}(U-u)\left(x_{i}\right)\right| & \leq C N^{-1}(\ln N)^{2}, \quad \text { if } 1-\tau_{L}<x_{i} \leq 1-\tau_{R}, \\
\sqrt{\frac{\varepsilon}{\theta}}\left|D^{-}(U-u)\left(x_{i}\right)\right| & \leq C N^{-1}(\ln N)^{2}, \quad \text { if } x_{i}>1-\tau_{R},
\end{aligned}
$$

where $\tau_{l}, \tau_{R}$ are defined in (9).

Proof. The proof is given in Appendix D.

\section{Global error bounds}

In this section, we examine the global accuracy of the linear interpolant

$$
\bar{U}(x):=\sum_{i=1}^{N-1} U\left(x_{i}\right) \phi_{i}(x), \quad x \in[0,1],
$$

where $\phi_{i}(x)$ is the standard piecewise linear basis functions, defined by the nodal values of $\phi_{i}\left(x_{k}\right)=\delta_{i, k}$. Note that

$$
(\bar{u}-u)^{\prime}(x)=D^{-} u\left(x_{i}\right)-u^{\prime}(x), \quad x \in\left(x_{i-1}, x_{i}\right] ;
$$

and, hence, we have the following bound on the linear interpolant $\bar{g}$ (for any $\left.g \in C^{1}\right)$ in the subinterval $I_{i}:=\left(x_{i-1}, x_{i}\right)$

$$
\begin{aligned}
\|g-\bar{g}\|_{I_{i}} & \leq C \min \left\{h_{i}^{2}\left\|g^{\prime \prime}\right\|_{I_{i}}, \int_{t=x_{i-1}}^{x_{i}}\left|g^{\prime}(t)\right| d t\right\} \\
\left\|(g-\bar{g})^{\prime}\right\|_{I_{i}} & \leq C \min \left\{h_{i}\left\|g^{\prime \prime}\right\|_{I_{i}},\left\|g^{\prime}\right\|_{I_{i}}\right\} .
\end{aligned}
$$

We conclude with the statement of the main result of this paper.

Theorem 7. If $u$ is the continuous solution of problem (1) and $U$ is the computed solution of $(11,8,13)$, then

$$
\begin{aligned}
\|u-\bar{U}\|_{1, \chi} & \leq C N^{-1} \ln N, \quad \text { assuming (12), } \\
\|u-\bar{U}\|_{1, \chi} & \leq C N^{-1}(\ln N)^{3}, \quad \text { not assuming (12); }
\end{aligned}
$$

where the norm $\|\cdot\|_{1, \chi}$ is defined in (10).

Proof. We only deal with the case where (12) applies. (See [13] for the other case). Using the decomposition $u=v+(u-v)(0) w_{L}+\left(u-v-w_{L}\right)(1) w_{R}$, splitting the argument to inside and outside the computational layer regions $\left[0, \sigma_{L}\right],\left[1-\sigma_{R}, 1\right]$, using the bounds $(32 \mathrm{a}),(7 \mathrm{~b})$ and $(7 \mathrm{c})$, we have the following interpolation error

$$
\|u-\bar{u}\| \leq C\left(N^{-1} \ln N\right)^{2} .
$$


We next want to estimate the global error in approximating the scaled flux. For the regular component it trivially follows that

$$
\left\|(v-\bar{v})^{\prime}\right\|_{I_{i}} \leq C N^{-1} .
$$

For the left layer component, we first consider the case where $\tau_{L} \leq \sigma_{L}$. By using the bound (32b), we can obtain

$$
\begin{array}{r}
\sqrt{\varepsilon \theta}\left\|\left(w_{L}-\bar{w}_{L}\right)^{\prime}\right\|_{I_{i}} \leq C N^{-1} \ln N, \quad \text { for } \quad x_{i} \leq \tau_{L}, \\
\left\|\left(w_{L}-\bar{w}_{L}\right)^{\prime}\right\|_{I_{i}} \leq C \frac{h_{L}}{\varepsilon \theta} e^{-\frac{\sqrt{\gamma \alpha}}{2 \sqrt{\varepsilon \theta}} \tau_{L}} \leq C N^{-1} \ln N, \quad \text { for } \quad \tau_{L}<x_{i} \leq \sigma_{L}, \\
\left\|\left(w_{L}-\bar{w}_{L}\right)^{\prime}\right\|_{I_{i}} \leq \frac{C}{\sqrt{\varepsilon \theta}} e^{-\frac{\sqrt{\gamma \alpha}}{4 \sqrt{\varepsilon \theta}} \sigma_{L}} e^{-\frac{\sqrt{\gamma \alpha}}{4 \sqrt{\varepsilon \theta}} \tau_{L}} \leq C N^{-1}, \quad \text { for } \quad x_{i}>\sigma_{L} .
\end{array}
$$

For the alternative case, where $\sigma_{L} \leq \tau_{L}$ we have the bounds

$$
\begin{array}{r}
\sqrt{\varepsilon \theta}\left\|\left(w_{L}-\bar{w}_{L}\right)^{\prime}\right\|_{I_{i}} \leq C N^{-1} \ln N, \quad \text { for } \quad x_{i} \leq \sigma_{L}, \\
\sqrt{\varepsilon \theta}\left\|\left(w_{L}-\bar{w}_{L}\right)^{\prime}\right\|_{I_{i}} \leq C e^{-\frac{\sqrt{\gamma \alpha}}{2 \sqrt{\varepsilon \theta}} \sigma_{L}} \leq C N^{-2}, \quad \text { for } \quad \sigma_{L}<x_{i} \leq \tau_{L}, \\
\left\|\left(w_{L}-\bar{w}_{L}\right)^{\prime}\right\|_{I_{i}} \leq \frac{C}{\sqrt{\varepsilon \theta}} e^{-\frac{\sqrt{\gamma \alpha}}{4 \sqrt{\varepsilon \theta}} \tau_{L}} e^{-\frac{\sqrt{\gamma \alpha}}{4 \sqrt{\varepsilon \theta}} \sigma_{L}} \leq C N^{-1}, \quad \text { for } \quad x_{i}>\tau_{L} .
\end{array}
$$

A similar argument is used to obtain the corresponding bounds for the right layer component (See [13] for details). From these bounds, we deduce that

$$
\|u-\bar{u}\|_{1, \chi} \leq C N^{-1} \ln N .
$$

Combining the interpolation bound (33) with the nodal error bound (31), we arrive at the following global error estimate:

$$
\|u-\bar{U}\| \leq C N^{-1} \ln N .
$$

Note also that $(\bar{U}-\bar{u})^{\prime}(x)=D^{-}(U-u)\left(x_{i}\right), \quad \forall x \in\left(x_{i-1}, x_{i}\right]$. Use this bound, Theorem 6 and the interpolation bound in (34) to complete the proof.

Remark 1. The error bounds in Theorem 7 extend to the case of $-1 \leq \mu<0$, when the first order operator $D^{-}$is replaced by $D^{+}$in the definition of $L^{N}$ and the definition of the piecewise uniform mesh is modified to

$$
\begin{gathered}
\rho_{L}^{-}:=\max \left\{1, \sqrt{\frac{\theta \gamma \alpha}{\varepsilon}}\right\} \quad \text { and } \quad \rho_{R}^{-}:=\max \left\{1, \frac{1}{2} \sqrt{\frac{\gamma \alpha}{\theta \varepsilon}}\right\} \\
\sigma_{L}^{-}:=\min \left\{\frac{1}{4}, \frac{4}{\rho_{L}^{-}} \ln N\right\} \quad \text { and } \quad \sigma_{R}^{-}:=\min \left\{\frac{1}{4}, \frac{2}{\rho_{R}^{-}} \ln N\right\} .
\end{gathered}
$$




\section{Numerical results}

Consider the following two parameter singularly perturbed problem

$$
-\varepsilon u^{\prime \prime}+\mu\left(1+x^{2}\right) u^{\prime}+(2-x) u=x^{3} ; u(0)=1, u(1)=0 .
$$

For this particular problem the parameters (1c) are $\alpha=1, \gamma=0.5$. Problem (35) is a variable coefficient problem, whose exact solution $u$ and it's first derivative $u^{\prime}$ were numerically approximated by applying the upwind finite difference (13a) on a fine piecewise-uniform Shishkin mesh $\Omega_{\text {fine }}(11),(11 \mathrm{~b})$ with $N=4096$. From this numerical solution, global approximations $u_{A}, u_{A}^{\prime}$ were generated using linear interpolation $\bar{U}$ and piecewise constant interpolation

$$
u_{A}^{\prime}(x):=D^{-} U\left(x_{k}\right), x \in\left(x_{k-1}, x_{k}\right], k=1, \ldots 4096 ; \quad u_{A}^{\prime}(0):=D^{-} U\left(x_{1}\right),
$$

respectively, over this fine Shishkin mesh.

Numerical approximations $U$ to the solution $u$ of (35) were subsequently generated over the parameter sets $S_{\varepsilon}:=\left\{2^{-2 j} ; j=0,1, \ldots, 30\right\}, S_{\mu}=\left\{2^{-2 j} ; j=\right.$ $0,1, \ldots, 10\}$ and a sequence of meshes $(11),(11 \mathrm{~b})$ with $N=\left\{2^{k} ; k=6,7, \ldots, 10\right\}$. For each set of parameters, global approximations $\bar{U}$ (to the solution $u$ of (35)) and $\overline{\left(D^{-} U\right)}$ to $u^{\prime}$ (the derivative of the solution of (35)) were generated using linear interpolation over the coarse mesh. For each particular triple $(\varepsilon, \mu, N)$ of parameter values, the global scaled $C^{1}$ error $\|u-\bar{U}\|_{1, \chi}$ (as defined in (10)) is estimated by calculating

$$
E_{\varepsilon, \mu}^{N}:=\left\|\chi\left(u_{A}^{\prime}-\overline{\left(D^{-} U\right)}\right)\right\|_{\Omega_{f i n e}}+\left\|u_{A}-\bar{U}\right\|_{\Omega_{f i n e}} .
$$

The results presented in Tables 1 and 2 display parameter-uniform convergence in the $\|\cdot\|_{1, \chi}$ norm.

For each $N$, the parameter-uniform orders of global convergence $p^{N}$ are estimated by computing

$$
E^{N}:=\max _{\mu \in S_{\mu}} E_{\mu}^{N}, E_{\mu}^{N}:=\max _{\varepsilon \in S_{\varepsilon}} E_{\varepsilon, \mu}^{N} \quad \text { and } \quad p^{N}:=\log _{2}\left(E^{N} / E^{2 N}\right),
$$

which are displayed in Table 3. For the particular test problem (35), these parameter-uniform orders of global convergence are higher than the theoretical rates established in Theorem 7. In [13], similiar numerical results for a constant coefficient two parameter problem are presented.

In [13], numerical results for a constant coefficient two parameter boundary value problem are seen to be also in line with the theoretical rates established in Theorem 7. In passing we note that the parameter-uniform convergence of the scaled discrete partial derivatives is also illustrated for variable coefficient elliptic and parabolic test problems, in the case of one parameter singularly perturbed partial differential equations, in $[5,6,7]$. 
Table 1: Computed global errors $E_{\varepsilon, 2^{-4}}^{N}$, where $\mu=2^{-4}$ and $\varepsilon$ varies

\begin{tabular}{|c|c|c|c|c|c|}
\hline$\epsilon / \mathrm{N}$ & 64 & 128 & 256 & 512 & 1024 \\
\hline $2^{0}$ & $1.42 \mathrm{e}-02$ & $7.07 \mathrm{e}-03$ & $3.44 \mathrm{e}-03$ & $1.61 \mathrm{e}-03$ & $6.90 \mathrm{e}-04$ \\
\hline $2^{-} 2$ & $5.29 \mathrm{e}-02$ & $2.65 \mathrm{e}-02$ & $1.29 \mathrm{e}-02$ & $6.05 \mathrm{e}-03$ & $2.60 \mathrm{e}-03$ \\
\hline $2^{-} 4$ & $6.23 \mathrm{e}-02$ & $3.02 \mathrm{e}-02$ & $1.45 \mathrm{e}-02$ & $6.74 \mathrm{e}-03$ & $2.88 \mathrm{e}-03$ \\
\hline $2^{-} 6$ & $1.53 \mathrm{e}-01$ & $7.94 \mathrm{e}-02$ & $3.96 \mathrm{e}-02$ & $1.87 \mathrm{e}-02$ & $8.09 \mathrm{e}-03$ \\
\hline $2^{-} 8$ & $4.42 \mathrm{e}-01$ & $2.56 \mathrm{e}-01$ & $1.36 \mathrm{e}-01$ & $6.70 \mathrm{e}-02$ & $2.95 \mathrm{e}-02$ \\
\hline $2^{-} 10$ & $\mathbf{8 . 5 8 e - 0 1}$ & $5.86 \mathrm{e}-01$ & $3.64 \mathrm{e}-01$ & $2.00 \mathrm{e}-01$ & $9.42 \mathrm{e}-02$ \\
\hline $2^{-} 12$ & $8.09 \mathrm{e}-01$ & $\mathbf{6 . 0 2 e - 0 1}$ & $\mathbf{4 . 1 4 e - 0 1}$ & $\mathbf{2 . 5 5 e - 0 1}$ & $\mathbf{1 . 3 4 e - 0 1}$ \\
\hline $2^{-} 14$ & $8.01 \mathrm{e}-01$ & $5.95 \mathrm{e}-01$ & $4.10 \mathrm{e}-01$ & $2.52 \mathrm{e}-01$ & $1.32 \mathrm{e}-01$ \\
\hline $2^{-} 16$ & $7.98 \mathrm{e}-01$ & $5.94 \mathrm{e}-01$ & $4.09 \mathrm{e}-01$ & $2.51 \mathrm{e}-01$ & $1.32 \mathrm{e}-01$ \\
\hline $2^{-} 18$ & $7.97 \mathrm{e}-01$ & $5.93 \mathrm{e}-01$ & $4.09 \mathrm{e}-01$ & $2.51 \mathrm{e}-01$ & $1.32 \mathrm{e}-01$ \\
\hline $2^{-} 40$ & $7.97 \mathrm{e}-01$ & $5.93 \mathrm{e}-01$ & $4.09 \mathrm{e}-01$ & $2.51 \mathrm{e}-01$ & $1.32 \mathrm{e}-01$ \\
\hline
\end{tabular}

Table 2: Computed maximum global errors $E_{\mu}^{N}$, in the scaled $C^{1}$ norm, measured over the set $S_{\varepsilon}=\left\{\varepsilon=2^{-2 j}, j=0,1 . ., 20\right\}$ for various values of $\mu$

\begin{tabular}{|c|c|c|c|c|c|}
\hline$\mu / \mathrm{N}$ & 64 & 128 & 256 & 512 & 1024 \\
\hline $2^{0}$ & $5.17 \mathrm{e}-01$ & $3.67 \mathrm{e}-01$ & $2.53 \mathrm{e}-01$ & $1.55 \mathrm{e}-01$ & $8.17 \mathrm{e}-02$ \\
\hline $2^{-2}$ & $4.59 \mathrm{e}-01$ & $3.27 \mathrm{e}-01$ & $2.25 \mathrm{e}-01$ & $1.38 \mathrm{e}-01$ & $7.27 \mathrm{e}-02$ \\
\hline $2^{-4}$ & $8.58 \mathrm{e}-01$ & $6.02 \mathrm{e}-01$ & $4.14 \mathrm{e}-01$ & $2.55 \mathrm{e}-01$ & $1.34 \mathrm{e}-01$ \\
\hline $2^{-6}$ & $1.27 \mathrm{e}+00$ & $8.72 \mathrm{e}-01$ & $5.56 \mathrm{e}-01$ & $3.42 \mathrm{e}-01$ & $1.80 \mathrm{e}-01$ \\
\hline $2^{-8}$ & $1.35 \mathrm{e}+00$ & $1.01 \mathrm{e}+00$ & $6.85 \mathrm{e}-01$ & $4.23 \mathrm{e}-01$ & $2.23 \mathrm{e}-01$ \\
\hline $2^{-10}$ & $1.38 \mathrm{e}+00$ & $1.03 \mathrm{e}+00$ & $7.01 \mathrm{e}-01$ & $4.33 \mathrm{e}-01$ & $2.28 \mathrm{e}-01$ \\
\hline $2^{-12}$ & $1.39 \mathrm{e}+00$ & $1.04 \mathrm{e}+00$ & $7.05 \mathrm{e}-01$ & $4.35 \mathrm{e}-01$ & $2.29 \mathrm{e}-01$ \\
\hline $2^{-14}$ & $1.39 \mathrm{e}+00$ & $1.04 \mathrm{e}+00$ & $7.06 \mathrm{e}-01$ & $4.36 \mathrm{e}-01$ & $2.30 \mathrm{e}-01$ \\
\hline$\cdot$ & $\cdot$ & $\cdot$ & $\cdot$ & $\cdot$ & $\cdot$ \\
\hline $2^{-20}$ & $1.39 \mathrm{e}+00$ & $1.04 \mathrm{e}+00$ & $7.07 \mathrm{e}-01$ & $4.36 \mathrm{e}-01$ & $2.30 \mathrm{e}-01$ \\
\hline
\end{tabular}

Table 3: Computed orders of parameter-uniform convergence $p^{N}$ in the scaled $C^{1}$ norm $\|\cdot\|_{1, \chi}$ (defined in (10))

\begin{tabular}{|c|c|c|c|c|}
\hline$N$ & 64 & 128 & 256 & 512 \\
\hline$p^{N}$ & 0.41 & 0.56 & 0.70 & 0.93 \\
\hline
\end{tabular}




\section{References}

[1] V. B. Andreev, Pointwise and weighted a priori estimates for the solution and its first derivative of a singularly perturbed convection-diffusion equation. Differ. Equ. 38 no. 7, (2002), 972-984.

[2] V. B. Andreev and I. A. Savin, The computation of boundary flow with uniform accuracy with respect to a small parameter. Comput. Math. Math. Phys. 36 no. 12, (1996), 1687-1692.

[3] P. A. Farrell, A. F. Hegarty, J. J. H. Miller, E. O'Riordan, G. I. Shishkin, Robust computational techniques for boundary layers, Chapman and Hall/CRC Press, Boca Raton, U.S.A., 2000.

[4] J. L. Gracia, E. O'Riordan and M. L. Pickett, A parameter robust higher order numerical method for a singularly perturbed two-parameter problem, Appl. Numer. Math., 56, (2006), 962-980.

[5] J. L. Gracia and E. O' Riordan, Numerical approximation of solution derivatives in the case of singularly perturbed time dependent reaction-diffusion problems, J. Comput. Appl. Math., 273, (2015), 13-24.

[6] J. L. Gracia and E. O' Riordan, Scaled discrete derivatives of singularly perturbed elliptic problems, Numer. Methods Partial Differential Equations, 31 no. 1, (2015), 225-252.

[7] J. L. Gracia and E. O' Riordan, Numerical approximation of solution derivatives of singularly perturbed parabolic problems of convection-diffusion type, Math. Comp., 85, (2016), 581-599.

[8] N. V. Kopteva and M. Stynes, Approximation of derivatives in a convectiondiffusion two-point boundary value problem, Appl. Numer. Math. 39 no. 1, (2001), 47-60.

[9] T. Linß, A posteriori error estimation for a singularly perturbed problem with two small parameters, Int. J. Numer. Anal. Model., 7 no. 3, (2010), 491-506.

[10] T. Linß and H.-G. Roos, Analysis of a finite-difference scheme for a singularly perturbed problem with two small parameters, J. Math. Anal. Appl. 289, (2004), 355-366.

[11] E. O'Riordan, M. L. Pickett and G. I. Shishkin, Singularly perturbed problems modeling reaction-convection-diffusion processes Comput. Methods Appl. Math., 3 no. 3, (2003), 424-442.

[12] E. O'Riordan, M. L. Pickett and G. I. Shishkin, Parameter-uniform finite difference schemes for singularly perturbed parabolic diffusion-convectionreaction problems, Math. Comp., 75, (2006), 1135-1154. 
[13] E. O'Riordan and M. L. Pickett, Numerical approximations to the scaled first derivatives of a two parameter singularly perturbed problem, ArXiv eprints, 1708.00682 [math.NA], (August, 2017).

[14] R. M. Priyadharshini and N. Ramanujam, Approximation of derivative to a singularly perturbed reaction-convection-diffusion problem with two parameters, J. Appl. Math. and Informatics, 27 no. 3-4, (2009), 517-529

[15] G. I. Shishkin, Approximations of solutions and derivatives for a singularly perturbed elliptic convection-diffusion equations, Math. Proc. Royal Irish Acad., 103A no. 2, (2003), 169-201.

[16] G. I. Shishkin, Discrete approximations of solutions and derivatives for a singularly perturbed parabolic convection-diffusion equation, J. Comput. Appl. Math., 166 no. 1, (2004), 247-266.

[17] H. Zarin, Exponentially graded mesh for a singularly perturbed problem with two small parameters, Appl. Numer. Math., 120, (2017), 233-242.

\section{Appendix A. Proof of Theorem 1.}

Proof. (i) In the reaction-diffusion case, where $\theta=1$, we decompose the regular component in a series of terms of increasing half powers of $\varepsilon$. That is, let

$$
\begin{aligned}
& v=\sum_{i=0}^{3} \varepsilon^{i / 2} v_{i}, \quad \text { where } \quad L_{0} v_{0}=f ; \quad \sqrt{\varepsilon} L_{0} v_{i}=\left(L_{0}-L_{\varepsilon, \mu}\right) v_{i-1}, i=1,2 ; \\
& \text { and } \quad \sqrt{\varepsilon} L_{\varepsilon, \mu} v_{3}=\left(L_{0}-L_{\varepsilon, \mu}\right) v_{2},
\end{aligned}
$$

For the convection-diffusion case, where $\theta>1$, we decompose the regular component in a series of terms of increasing integer powers of $\varepsilon$ as follows:

$$
\begin{aligned}
& v=\sum_{i=0}^{3} \varepsilon^{i} v_{i}, \quad \text { where } \quad L_{\mu} v_{0}=f ; \quad \sqrt{\varepsilon} L_{\mu} v_{i}=\left(L_{\mu}-L_{\varepsilon, \mu}\right) v_{i-1}, i=1,2 ; \\
& \text { and } \quad \sqrt{\varepsilon} L_{\varepsilon, \mu} v_{3}=\left(L_{\mu}-L_{\varepsilon, \mu}\right) v_{2}, \quad v_{2}(0)=v_{3}(0)=v_{3}(1)=0
\end{aligned}
$$

and $v_{0}(0), v_{1}(0)$ are suitably chosen. All of the bounds (6) are then established using the argument in [4]. More details are available in [13].

(ii) Observe that

$$
\begin{aligned}
L_{\varepsilon, \mu} e^{-\frac{\sqrt{\gamma \alpha}}{2 \sqrt{\varepsilon \theta}} x} & =a\left(\frac{b}{a}-\frac{1}{a} \frac{\gamma \alpha}{4 \theta}-\frac{\mu}{2} \sqrt{\frac{\gamma \alpha}{\varepsilon \theta}}\right) e^{-\frac{\sqrt{\gamma \alpha}}{2 \sqrt{\varepsilon \theta}} x} \\
& \geq a \gamma\left(1-\frac{1}{4 \theta}-\frac{1}{2} \sqrt{\frac{\mu^{2} \alpha}{\gamma \varepsilon \theta}}\right) e^{-\frac{\sqrt{\gamma \alpha}}{2 \sqrt{\varepsilon \theta}} x} \geq 0 ; \\
\text { and } L_{\varepsilon, \mu} e^{-\frac{\sqrt{\gamma \alpha \theta}}{\sqrt{\varepsilon}}(1-x)} & =a\left(\frac{b}{a}-\frac{1}{a} \gamma \alpha \theta+\mu \sqrt{\frac{\gamma \alpha \theta}{\varepsilon}}\right) e^{-\frac{\sqrt{\gamma \alpha \theta}}{\sqrt{\varepsilon}}(1-x)} \geq 0 .
\end{aligned}
$$

The comparison principle then yields the pointwise bounds (7a). 
(iii) From the bounds (4) established in Lemma 1, we deduce the following derivative bounds on the singular components $w_{L}, w_{R}$. For $0 \leq k \leq 5$,

$$
\left|w_{L}\right|_{k},\left|w_{R}\right|_{k} \leq C\left(\sqrt{\frac{\theta}{\varepsilon}}\right)^{k}
$$

When $\theta>1$, we can derive sharper bounds on the derivatives of $w_{L}$ by introducing the secondary decomposition

$$
\begin{aligned}
& w_{L}=\sum_{i=0}^{3} \varepsilon^{i} w_{i}, \quad \text { where } \quad L_{\mu} w_{0}=0, w_{0}(0)=1 ; \\
& \quad \varepsilon L_{\mu} w_{i}=\left(L_{\mu}-L_{\varepsilon, \mu}\right) w_{i-1}, \quad w_{i}(0)=0, i=1,2 ; \\
& \text { and } \quad \varepsilon L_{\varepsilon, \mu} w_{3}=\left(L_{\mu}-L_{\varepsilon, \mu}\right) w_{2}, \quad w_{3}(0)=w_{3}(1)=0 .
\end{aligned}
$$

Observe that $w_{L}(1)=w_{0}(1)+\varepsilon w_{1}(1)+\varepsilon^{2} w_{2}(1) \neq 0$. From this expansion one can deduce that

$$
\left|w_{L}(1)\right| \leq e^{-\frac{\gamma}{\mu}}, \quad\left|w_{L}\right|_{k} \leq C \mu^{-k}, 1 \leq k \leq 5 .
$$

Hence, we have deduced that

$$
\left|w_{L}\right|_{k} \leq C\left(\frac{1}{\sqrt{\varepsilon \theta}}\right)^{k}, \quad 1 \leq k \leq 5 .
$$

Our next step in the proof is to deduce (7b). For those points within the right layer, we have that

$$
e^{-\frac{\sqrt{\gamma \alpha \theta}}{\sqrt{\varepsilon}}(1-x)} \geq C, 1-\sqrt{\frac{\varepsilon}{\theta}} \leq x \leq 1
$$

and so

$$
\left|w_{R}(x)\right|_{k} \leq C\left(\sqrt{\frac{\theta}{\varepsilon}}\right)^{k} \leq C\left(\sqrt{\frac{\theta}{\varepsilon}}\right)^{k} e^{-\frac{\sqrt{\gamma \alpha \theta}}{\sqrt{\varepsilon}}(1-x)}, 1-\sqrt{\frac{\varepsilon}{\theta}} \leq x \leq 1 .
$$

Now we return to the argument from Lemma 1 . If $x<1-\sqrt{\frac{\varepsilon}{\theta}}$, construct a neighbourhood $N_{x}=(p-r, p)$ so that $x \in N_{x}$. Then there exists a $y \in N_{x}$ such that

$$
\begin{gathered}
\left|w_{R}^{\prime}(y)\right| \leq \frac{2\left\|w_{R}\right\|_{N_{x}}}{r} . \\
w_{R}^{\prime}(x)=w_{R}^{\prime}(y)+\int_{t=y}^{x} w_{R}^{\prime \prime} d t=w_{R}^{\prime}(y)+\frac{1}{\varepsilon} \int_{t=y}^{x} \mu a w_{R}^{\prime}+b w_{R} d t \\
=w_{R}^{\prime}(y)+\frac{\mu}{\varepsilon}\left(\left(a w_{R}\right)(x)-\left(a w_{R}\right)(y)\right)-\frac{1}{\varepsilon} \int_{t=y}^{x} \mu a^{\prime} w_{R}-b w_{R} d t .
\end{gathered}
$$

Thus

$$
\left|w_{R}^{\prime}(x)\right| \leq C\left(\frac{1}{r}+\frac{\mu}{\varepsilon}+\frac{r}{\varepsilon}\right)\left\|w_{R}\right\|_{N_{x}} \leq C\left(\frac{1}{r}+\frac{\mu}{\varepsilon}+\frac{r}{\varepsilon}\right) e^{-\frac{\sqrt{\gamma \alpha \theta}}{\sqrt{\varepsilon}}(1-x)} e^{\frac{\sqrt{\gamma \alpha \theta}}{\sqrt{\varepsilon}} r} .
$$

By taking

$$
r=\sqrt{\frac{\varepsilon}{2 \theta}}
$$

we deduce

$$
\left|w_{R}^{\prime}(x)\right| \leq C \sqrt{\frac{\theta}{\varepsilon}} e^{-\frac{\sqrt{\gamma \alpha \theta}}{\sqrt{\varepsilon}}(1-x)}
$$


From the differential equation defining $w_{R}, \varepsilon\left|w_{R}^{\prime \prime}(x)\right| \leq C \sqrt{\theta \varepsilon}\left|w_{R}^{\prime}(x)\right|+C\left|w_{R}(x)\right|$, which will establish the bound on the second order derivative of $w_{R}$. Use the bounds in Lemma 1, to establish the bounds (7b) on the higher derivatives of $w_{R}(x)$.

(iv) To complete the proof, we establish the bound (7c). For the case of $\theta=1$, the above argument (used to establish $(7 \mathrm{~b})$ ) can be repeated (with $1-x$ replaced by $x$ ). In the other case of $\theta>1$, we use the decomposition (37). Observe that for $0 \leq k \leq 5$,

$$
\left|w_{i}(x)\right|_{k} \leq \frac{C}{\mu^{2 i+k}} e^{-\gamma x / \mu}, \quad i=0,1,2
$$

and hence, using a maximum principle for the second order operator $L_{\varepsilon, \mu}$ we have

$$
\left|w_{3}(x)\right| \leq \frac{C}{\mu^{6}} e^{-\frac{\gamma x}{2 \mu}}
$$

Now repeat the argument used to establish (7b) (taking $r=\mu$ ) to deduce that for $x>\mu$,

$$
\left|w_{3}^{\prime}(x)\right| \leq \frac{C}{\mu^{7}}(1+\theta) e^{-\frac{\gamma x}{2 \mu}} \quad \text { and } \quad\left|w_{3}^{\prime \prime}(x)\right| \leq \frac{C}{\mu^{8}}\left(1+\theta+\theta^{2}\right) e^{-\frac{\gamma x}{2 \mu}}
$$

Hence, since we are in the case of $\varepsilon \leq C \mu^{2}$,

$$
\begin{aligned}
\left|w_{L}^{\prime}(x)\right| & \leq \frac{C}{\mu}\left(1+\frac{\varepsilon}{\mu^{2}}+\frac{\varepsilon^{2}}{\mu^{4}}+\frac{\varepsilon^{3}}{\mu^{6}}\left(1+\frac{\mu^{2}}{\varepsilon}\right)\right) e^{-\frac{\gamma x}{2 \mu}} \leq \frac{C}{\mu} e^{-\frac{\gamma x}{2 \mu}}, \\
\left|w_{L}(x)\right|_{k} & \leq \frac{C}{\mu^{k}} e^{-\frac{\gamma x}{2 \mu}}, \quad k=2,3 .
\end{aligned}
$$

Continuing this argument for the higher derivatives establishes (7c) for $\theta>1$.

\section{Appendix B. Proof of Lemma 4.}

Proof. Using the bounds (6) on the derivatives of the regular component $v$, we have

$$
\left|L^{N}(V-v)\left(x_{i}\right)\right| \leq \begin{cases}C(\varepsilon+\mu) N^{-1}, & \text { if } x_{i}=\sigma_{L}, 1-\sigma_{R} \\ C\left(\sqrt{\varepsilon \theta} N^{-1}+\mu\right) N^{-1} & \text { otherwise }\end{cases}
$$

(i) Looking first to establish a bound at the end-point $x=0$, if $\theta>1$, consider the linear barrier function

$$
B\left(x_{i}\right):=C_{1}\left(\frac{\varepsilon}{\mu}+1\right) N^{-1} x_{i} .
$$

Observe that $L^{N}\left(B\left(x_{i}\right) \pm(V-v)\left(x_{i}\right)\right) \geq 0$ for $C_{1}$ large enough. Applying the discrete minimum principle and using $\theta=\frac{\alpha \mu^{2}}{\gamma \varepsilon}>1$ we deduce that

$$
\left|(V-v)\left(x_{i}\right)\right| \leq C N^{-1}\left(\frac{\varepsilon}{\mu}\right) x_{i} \leq C N^{-1} \mu x_{i},
$$

yielding the bound $\left|D^{+}(V-v)(0)\right| \leq C \mu N^{-1}$.

(ii) In the reaction-diffusion case (where $\theta=1$ ) consider the barrier function

$$
B_{2}\left(x_{i}\right):=C_{1}\left(\sqrt{\varepsilon} N^{-2} \ln N R_{1}\left(x_{i}\right)\right)+C_{2}\left(N^{-2} \frac{\sqrt{\varepsilon}}{\beta}+N^{-1} x_{i}\right),
$$


where the wedge function $R_{1}\left(x_{i}\right)$ is defined by

$$
R_{1}\left(x_{i}\right):= \begin{cases}\frac{x_{i}}{\sigma_{L}}, & \text { if } x_{i} \leq \sigma_{L} \\ 1, & \text { if } \sigma_{L}<x_{i}<1-\sigma_{R} \\ \frac{1-x_{i}}{\sigma_{R}}, & \text { if } x_{i} \geq 1-\sigma_{R}\end{cases}
$$

We find that

$$
L^{N} R_{1}\left(x_{i}\right) \geq \begin{cases}0, & \text { if } x_{i}<\sigma_{L} \\ \frac{\varepsilon N}{\sigma_{L}}+\frac{\mu \alpha}{\sigma_{L}}, & \text { if } x_{i}=\sigma_{L} \\ 0, & \text { if } \sigma_{L}<x_{i}<\sigma_{R} \\ \frac{\varepsilon N}{\sigma_{R}}, & \text { if } x_{i}=1-\sigma_{R} \\ -\frac{\mu a}{\sigma_{R}}, & \text { if } x_{i}>1-\sigma_{R} .\end{cases}
$$

Since

$$
L^{N}\left(N^{-2} \frac{\sqrt{\varepsilon}}{\beta}+N^{-1} x_{i}\right) \geq a \mu N^{-1}+N^{-2} \sqrt{\varepsilon}+N^{-1} x_{i} b \geq C N^{-1}\left(\mu+N^{-1} \sqrt{\varepsilon}\right),
$$

we see that

$L^{N} B_{2}\left(x_{i}\right) \geq\left\{\begin{array}{l}C N^{-1}\left(\mu+N^{-1} \sqrt{\varepsilon}\right), \quad \text { if } x_{i}<\sigma_{L}, \sigma_{L}<x_{i}<\sigma_{R} \\ C N^{-1}\left(\sqrt{\varepsilon} N^{-1} \ln N\left(\frac{\varepsilon N}{\sigma_{L}}\right)+\mu\right), \quad \text { if } x_{i}=\sigma_{L} \\ C N^{-1}\left(\sqrt{\varepsilon} N^{-1} \ln N\left(\frac{\varepsilon N}{\sigma_{R}}\right)+\mu\right), \quad \text { if } x_{i}=1-\sigma_{R}, \\ C_{1} N^{-1}\left(\sqrt{\varepsilon} N^{-1} \ln N\left(-\frac{\mu a}{\sigma_{R}}\right)\right)+C_{2} N^{-1}\left(\mu+N^{-1} \sqrt{\varepsilon}\right), \text { if } x_{i}>1-\sigma_{R} .\end{array}\right.$

Now when $\theta=1$, for the bound at the transitions points, note that

$$
\sqrt{\varepsilon} N^{-2} \ln N\left(\frac{\varepsilon N}{\sigma_{L}}\right)=\sqrt{\varepsilon} N^{-2} \ln N\left(\frac{\varepsilon N}{\sigma_{R}}\right)=\frac{N^{-1} \varepsilon \sqrt{\gamma \alpha}}{4} .
$$

Also for $C_{2}$ sufficiently large, for the bound in the layer region near $x=1$,

$$
C_{1}\left(\sqrt{\varepsilon} N^{-2} \ln N\left(-\frac{\mu a}{\sigma_{R}}\right)+C_{2}\left(\mu N^{-1}\right) \geq C_{3} \mu N^{-1}\right.
$$

We therefore have deduced that $\left|(V-v)\left(x_{i}\right)\right| \leq B_{2}\left(x_{i}\right)$. Using $\sigma_{L}=C \sqrt{\varepsilon} \ln N$ we see that

$$
\left|(V-v)\left(h_{L}\right)\right| \leq C N^{-1}\left(N^{-1} h_{L}+N^{-1} \sqrt{\varepsilon}+h_{L}\right),
$$

which yields the bound

$$
\left|D^{+}(V-v)(0)\right| \leq C N^{-1}\left(N^{-1}+N^{-1} \frac{\sqrt{\varepsilon}}{h_{L}}+1\right) \leq C N^{-1} .
$$

Hence, for both cases, we have established the bound at the left end-point $x=0$.

(iii) For the other end of the interval with $x=1$, consider the case of $\theta>1$ and the barrier function

$$
B_{3}\left(x_{i}\right):=C_{1}\left(\frac{\varepsilon}{\mu}+1\right) N^{-1}\left(x_{i}-1+\tilde{\psi}\left(x_{i}\right)\right)
$$

where the mesh function $\tilde{\psi}\left(x_{i}\right)$ satisfies

$$
-\varepsilon \delta^{2} \tilde{\psi}+C_{*} \sqrt{\varepsilon \theta} D^{-} \tilde{\psi}=0, x_{i} \in(0,1), \tilde{\psi}(0)=1, \tilde{\psi}(1)=0 ; C_{*}:=A \sqrt{\frac{\gamma}{\alpha}} .
$$


Compare this barrier function to the barrier function used at the start of Lemma 3. Applying the discrete maximum principle and using $\theta>1$ it follows that

$$
\left|(V-v)\left(x_{i}\right)\right| \leq C N^{-1}\left(x_{i}-1+\tilde{\psi}\left(x_{i}\right)\right)
$$

In order to use this to find a bound on $D^{+}(V-v)(1)$ we need to bound $D^{-} \tilde{\psi}(1)$. Defining $F_{i}:=D^{-} \tilde{\psi}\left(x_{i}\right)$, using (40) we see that

$$
-\varepsilon\left(\frac{F_{i+1}-F_{i}}{\bar{h}_{i}}\right)+C_{*} \sqrt{\varepsilon \theta} F_{i}=0, \quad x_{i} \in(0,1) .
$$

For $N$ large enough we conclude that

$$
D^{-} \tilde{\psi}(1)=\left|F_{N}\right| \leq C \sqrt{\frac{\theta}{\varepsilon}} .
$$

Using this bound and $(V-v)(1)=0$, we can deduce the bound (see [13] for details)

$$
\left|D^{-}(V-v)(1)\right| \leq C\left(N^{-1}\left(1+\left|D^{-} \tilde{\psi}(1)\right|\right)\right) \leq C N^{-1}\left(1+\sqrt{\frac{\theta}{\varepsilon}}\right) .
$$

This yields the desired bound at $x=1$ in the convection-diffusion case where $\theta>1$.

(iv) For the reaction-diffusion case, where $\theta=1$ the argument is more complicated. Consider $B_{4}\left(x_{i}\right):=C_{1}\left(\sqrt{\varepsilon} N^{-2} \ln N\right) R_{1}\left(x_{i}\right)+C_{2}\left(N^{-2} \frac{\sqrt{\varepsilon}}{\beta}+N^{-1}\left(x_{i}-1+\tilde{\psi}\left(x_{i}\right)\right)\right.$ with $R_{1}, \tilde{\psi}$ are as defined previously in (38) and (40) respectively. This fourth barrier function is a minor alteration to the barrier function $B_{2}\left(x_{i}\right)$. We can show that

$$
L^{N}\left(N^{-2} \frac{\sqrt{\varepsilon}}{\beta}+N^{-1}\left(x_{i}-1+\psi\left(x_{i}\right)\right) \geq C N^{-1}\left(\mu+N^{-1} \sqrt{\varepsilon}\right)\right.
$$

and using (39) we see that

$$
L^{N} B_{4}\left(x_{i}\right) \geq\left\{\begin{array}{l}
C N^{-1}\left(\mu+N^{-1} \sqrt{\varepsilon}\right), \quad \text { if } x_{i}<\sigma_{L}, \sigma_{L}<x_{i}<\sigma_{R} \\
C N^{-1}\left(\sqrt{\varepsilon} N^{-1} \ln N\left(\frac{\varepsilon N}{\sigma_{L}}\right)+\mu\right), \quad \text { if } x_{i}=\sigma_{L}, 1-\sigma_{R} \\
C_{1}\left(\sqrt{\varepsilon} N^{-2} \ln N\left(-\frac{\mu a}{\sigma_{R}}\right)+C_{2} N^{-1}\left(\mu+N^{-1} \sqrt{\varepsilon}\right), \quad \text { if } x_{i}>1-\sigma_{R}\right.
\end{array}\right.
$$

As before, as $\theta=1$,

$$
\sqrt{\varepsilon} N^{-2} \ln N\left(\frac{\varepsilon N}{\sigma_{L}}\right)=\sqrt{\varepsilon} N^{-2} \ln N\left(\frac{\varepsilon N}{\sigma_{R}}\right)=\frac{N^{-1} \varepsilon \sqrt{\gamma \alpha}}{4} ;
$$

and also for $C_{2}$ sufficiently large

$$
C_{1}\left(\sqrt{\varepsilon} N^{-2} \ln N\left(-\frac{\mu a}{\sigma_{R}}\right)+C_{2}\left(\mu N^{-1}\right) \geq C_{3} \mu N^{-1} .\right.
$$

We therefore have

$$
L^{N} B_{4}\left(x_{i}\right) \geq\left\{\begin{array}{l}
C N^{-1}\left(\mu+N^{-1} \sqrt{\varepsilon}\right), \quad \text { if } x_{i}<\sigma_{L}, \sigma_{L}<x_{i}<\sigma_{R}, \\
C N^{-1}(\mu+\varepsilon), \quad \text { if } x_{i}=\sigma_{L}, 1-\sigma_{R}, \\
C_{1} N^{-1} \sqrt{\varepsilon} N^{-1} \ln N\left(-\frac{\mu a}{\sigma_{R}}\right)+C_{2} N^{-1}\left(\mu+N^{-1} \sqrt{\varepsilon}\right), \text { if } x_{i}>1-\sigma_{R} .
\end{array}\right.
$$

Using the discrete maximum principle we deduce that

$$
\left|(V-v)\left(x_{i}\right)\right| \leq C_{1}\left(\sqrt{\varepsilon} N^{-2} \ln N R_{1}\right)+C_{2}\left(N^{-2} \frac{\sqrt{\varepsilon}}{\beta}+N^{-1}\left(x_{i}-1+\tilde{\psi}\left(x_{i}\right)\right),\right.
$$


which yields the bound

$$
\left.\left|\mathcal{V}_{N}\right| \leq C_{1}\left(\frac{\sqrt{\varepsilon} N^{-2} \ln N\left(\frac{h_{R}}{\sigma_{R}}\right)}{h_{R}}\right)+C_{2}\left(\frac{N^{-2} \sqrt{\varepsilon}}{\beta h_{R}}+\frac{N^{-1} h_{R}}{h_{R}}+N^{-1}\left|D^{-} \tilde{\psi}(1)\right|\right)\right) .
$$

Simplifying we have

$$
\left|D^{-}(V-v)(1)\right| \leq C N^{-1}\left(1+\sqrt{\frac{\theta}{\varepsilon}}\right)
$$

which completes the proof.

\section{Appendix C. Proof of Theorem 5.}

Proof. (i) At the interior points, using the truncation error bounds (23), we can establish that

$$
\left|\hat{L}^{N} D^{-}(V-v)\left(x_{i}\right)\right| \leq \begin{cases}C N^{-1}, & \text { if } x_{i} \neq \sigma_{L}+H, \sigma_{L}, 1-\sigma_{R}, 1-\sigma_{R}+h_{R}, \\ C\left(\frac{\sqrt{\varepsilon}}{\sqrt{\theta} \ln N}+N^{-1}\right), & \text { if } x_{i}=\sigma_{L}, \\ C\left(\varepsilon+\mu+N^{-1}\right), & \text { if } x_{i}=\sigma_{L}+H, \\ C\left(\varepsilon+N^{-1}\right), & \text { if } x_{i}=1-\sigma_{R}, \\ C\left(\frac{\sqrt{\varepsilon \theta}+\sqrt{\frac{\theta \mu^{2}}{\varepsilon}}}{\ln N}+N^{-1}\right), & \text { if } x_{i}=1-\sigma_{R}+h_{R},\end{cases}
$$

We next define a combination of barrier functions, which allow us to bound

$$
\sqrt{\frac{\varepsilon}{\theta}}\left|D^{-}(V-v)\left(x_{i}\right)\right|
$$

This initial set of barrier functions are linear and step functions. In order to establish the sharper bounds on $\left|D^{-}(V-v)\left(x_{i}\right)\right|$ these barrier functions are replaced by discrete exponential barrier functions. Define the following ramp functions

$$
R_{2}\left(x_{i}\right):=\left\{\begin{array}{ll}
\frac{x_{i}}{\sigma_{L}}, & \text { if } x_{i} \leq \sigma_{L}, \\
1, & \text { if } \sigma_{L}<x_{i} \leq 1
\end{array} \quad R_{3}\left(x_{i}\right):= \begin{cases}\frac{x_{i}}{1-\sigma_{R}}, & \text { if } x_{i} \leq 1-\sigma_{R} \\
1, & \text { if } 1-\sigma_{R}<x_{i} \leq 1\end{cases}\right.
$$

and step functions

$$
S_{1}\left(x_{i}\right):=\left\{\begin{array}{ll}
0, & \text { if } x_{i} \leq \sigma_{L}, \\
1, & \text { if } \sigma_{L}<x_{i} \leq 1
\end{array} \quad S_{2}\left(x_{i}\right):= \begin{cases}0, & \text { if } x_{i} \leq 1-\sigma_{R} \\
1, & \text { if } 1-\sigma_{R}<x_{i} \leq 1\end{cases}\right.
$$

Consider the barrier function

$$
\begin{array}{r}
B_{5}\left(x_{i}\right):=N^{-1}\left(C_{1} \sqrt{\varepsilon \theta} \ln N R_{2}+C_{2}\left(1+\frac{\mu}{\varepsilon}\right)\left(S_{1} N^{-1}+4 R_{2}\right)+C_{3}\left(R_{2}+R_{3}\right)\right) \\
+N^{-1}\left(C_{4}\left(1+\frac{\mu}{\varepsilon}\right)\left(N^{-1} S_{2}+2\left(R_{2}+R_{3}\right)\right)+C_{5}\right) .
\end{array}
$$

We find that $\hat{L}^{N}\left(B_{5} \pm \mathscr{V}_{i}^{-}\right) \geq 0$ (see [13] for details). Applying the maximum principle we get derivative bounds with scaling everywhere. That is, we have established the error bound

$$
\sqrt{\frac{\varepsilon}{\theta}}\left|D^{-}(V-v)\left(x_{i}\right)\right| \leq C N^{-1} \ln N, \quad x_{i} \in(0,1] .
$$

We now proceed to improve on this error bound. 
(ii) Consider first the case of $\theta=1$. Instead of using barrier functions involving ramps to deal with the truncation error at $x_{i}=\sigma_{L}+H$ and $x_{i}=1-\sigma_{r}+h_{r}$ we define the following two mesh functions

$$
\begin{aligned}
& Z_{L}\left(x_{i}\right):= \begin{cases}\left(1+\rho_{L} H\right)^{-1}\left(1+\rho_{L} h_{L}\right)^{i-\frac{N}{4}-1,} & \text { if } x_{i} \leq \sigma_{L}, \\
1, & \text { if } \sigma_{L}<x_{i} \leq 1\end{cases} \\
& Z_{R}\left(x_{i}\right):= \begin{cases}1, & \text { if } x_{i} \leq 1-\sigma_{R} \\
\left(1+0.5 \rho_{R} h_{R}\right)^{\frac{3 N}{4}+1-i}, & \text { if } x_{i} \geq 1-\sigma_{R}+h_{R},\end{cases}
\end{aligned}
$$

Consider the barrier function

$$
\begin{aligned}
B_{6}\left(x_{i}\right):=N^{-1}\left(C_{1} \sqrt{\varepsilon}\right. & \left.\ln N R_{2}+C_{2}\left(Z_{L}+\ln N R_{2}\right)+C_{3}\left(N^{-1} S_{1}+\frac{R_{2}}{4}\right)\right) \\
& +C_{4} N^{-1}\left(\left(R_{2}+R_{3}\right)+C_{5}\left(\sqrt{\varepsilon}+\frac{\mu}{\sqrt{\varepsilon}}\right) Z_{R}+C_{6}\right)
\end{aligned}
$$

and use the maximum principle (see [13] for details) to deduce that

$$
\left|D^{-}(V-v)\left(x_{i}\right)\right| \leq C N^{-1}(\ln N), \quad \text { if } 0<x_{i} \leq 1 ; \quad \text { and } \theta=1 .
$$

(iii) Next consider the case of $\theta>1$. Define the following three mesh functions;

$$
\begin{gathered}
P\left(x_{i}\right):= \begin{cases}0, & \text { if } x_{i} \leq \sigma_{L} \\
\left(1+0.5 \rho_{R} H\right)^{i-\frac{3 N}{4},} & \text { if } \sigma_{L}+H \leq x_{i} \leq 1-\sigma_{R}, \\
\left(1+\frac{\rho_{R} h_{R}}{32}\right)^{i-3 N / 4-1}, & \text { if } 1-\sigma_{R}+h_{R} \leq x_{i} \leq 1 .\end{cases} \\
Q\left(x_{i}\right):= \begin{cases}0, & \text { if } x_{i} \leq 1-\sigma_{R}, \\
\left(1+\frac{\rho h_{R}}{32}\right)^{i-3 N / 4-1}, & \text { if } 1-\sigma_{R}+h_{R} \leq x_{i} \leq 1 .\end{cases} \\
\hat{Z}_{L}\left(x_{i}\right):=\left\{\begin{array}{ll}
\left(1+\frac{\rho_{R} H}{4}\right)^{-1}\left(1+0.5 \rho_{R} h_{L}\right)^{i-\frac{N}{4}-1}, & \text { if } x_{i} \leq \sigma_{L}, \\
1, & \text { if } \sigma_{L}<x_{i} \leq 1
\end{array} .\right.
\end{gathered}
$$

Considering the linear combination

$$
\Psi\left(x_{i}\right)=N^{-1}\left(\left(1+\frac{\mu}{\varepsilon}\right) N^{-1} Q+\frac{8}{\alpha}\left(\frac{\varepsilon}{\mu}+1\right) P+\frac{128}{\gamma^{2} \alpha}(\mu+\varepsilon) \ln N R_{2}\right),
$$

we see that (see [13] for details)

$$
\hat{L}^{N}\left(\Psi\left(x_{i}\right)\right) \geq \begin{cases}0, & \text { if } x_{i} \neq 1-\sigma_{R}+h_{R}, \\ \frac{C}{\ln N}\left(\mu+\frac{\mu^{2}}{\varepsilon}\right), & \text { if } x_{i}=1-\sigma_{R}+h_{R} .\end{cases}
$$

Use the discrete maximum principle to derive the bound

$\left|\left(D^{-}(V-v)\left(x_{i}\right)\right)\right| \leq N^{-1}\left(C_{1} \mu \ln N R_{2}+C_{2}\left(\frac{\varepsilon}{\mu}+1\right) \hat{Z}_{L}+C_{3}\left(R_{2}+R_{3}\right)+C_{4} N \Psi+C_{5}\right)$.

If $x_{i} \leq 1-\sigma_{R}$ we have established the bound $\left|\left(D^{-}(V-v)\left(x_{i}\right)\right)\right| \leq C N^{-1}$; and we have removed all scaling outside the computational layer region on the right. 


\section{Appendix D: Proof of Theorem 6}

Proof. (i) We begin by examining the error in the layer function $W_{L}\left(W_{R}\right)$ in the fine mesh region on the right-hand (left-hand) side of the domain. Let us first consider the error $W_{L}-w_{L}$ in the right layer region $\left(1-\sigma_{R}, 1-\tau_{R}\right)$. For $x \geq 0.5$ and $x_{i} \geq 0.5$

$$
\begin{aligned}
\left|w_{L}(x)\right| & \leq C e^{-2 \rho_{L} \sigma_{L}} e^{-\rho_{L}\left(x-2 \sigma_{L}\right)} \leq C N^{-4} ; \\
\left|W_{L}\left(x_{i}\right)\right| & \leq C\left(1+\rho_{L} h_{L}\right)^{-N / 2} \leq C N^{-4}, \quad \text { as } \quad H \geq h_{L} .
\end{aligned}
$$

If $\tau_{R} \leq \sigma_{R}$, then $\sqrt{\frac{\theta}{\varepsilon}} \leq C N^{2}$ and so

$$
\left|\left(w_{L}-W_{L}\right)\left(x_{i}\right)\right| \leq C N^{-4} \leq C N^{-2} \sqrt{\frac{\varepsilon}{\theta}}, \quad \text { if } \quad x_{i} \geq 0.5
$$

Hence, $\left|D^{-}\left(w_{L}-W_{L}\right)\left(x_{i}\right)\right| \leq C N^{-1}$, if $x_{i} \geq 0.5$ and $\tau_{R} \leq \sigma_{R}$. An analogous argument can be used to establish $\left|D^{-}\left(w_{R}-W_{R}\right)\left(x_{i}\right)\right| \leq C N^{-2}$, if $x_{i} \geq 0.5$ and $\tau_{L} \leq \sigma_{L}$.

(ii) Consider the left layer error $D^{-}\left(W_{L}-w_{L}\right)$ in the region $\left[0, \sigma_{L}+\bar{H}\right]$. A more refined analysis (to that used in Theorem 3)) is required. The analysis requires the construction of a discrete barrier function across the non-uniform mesh and using a sharper truncation error analysis. Using the truncation error bounds (23) and the exponential bounds in Theorem 1 in the region $\left(0, \sigma_{L}+H\right)$, we have

$$
\begin{aligned}
\left|\hat{L}^{N} D^{-}\left(W_{L}-w_{L}\right)\left(x_{i}\right)\right| & \leq \frac{C}{\sqrt{\varepsilon \theta}} N^{-1} \ln N e^{-\rho_{L} x_{i}}, \quad x_{i}<\sigma_{L} \\
\left|\hat{L}^{N} D^{-}\left(W_{L}-w_{L}\right)\left(x_{i}\right)\right| & \leq C\left(\frac{1}{\varepsilon \theta^{2}}+\frac{\mu}{\varepsilon \theta} N^{-1} \ln N\right) e^{-\rho_{L} x_{i}}, \quad x_{i}=\sigma_{L} .
\end{aligned}
$$

We now construct a suitable barrier function (which is similar to $\Psi_{L}$ defined in (15a)):

$$
\Psi_{1}\left(x_{i}\right):=\left(1+0.5 \rho_{L} h_{L}\right)^{-i}, 0 \leq x_{i} \leq \sigma_{L} ; \quad \Psi_{1}\left(\sigma_{L}+H\right):=0 .
$$

For $x_{i}<\sigma_{L}$, as in Theorem $2, \hat{L}^{N} \Psi_{1}\left(x_{i}\right) \geq C e^{-0.5 \rho_{L} x_{i}}$ and for $x_{i}=\sigma_{L}$, using (19) and (2), (see [13] for details)

$$
\hat{L}^{N} \Psi_{1}\left(\sigma_{L}\right) \geq \frac{\varepsilon}{2 h_{L} \bar{h}_{L}}\left(1-\frac{\rho_{L}}{N}\left(1-\sigma_{R}\right)+1-\frac{2 \ln N}{N}\right) \Psi_{1}\left(\sigma_{L}\right)
$$

for $N$ sufficiently large. In the case where $\tau_{L} \leq \sigma_{L}$, then $\rho_{L} \leq N$ and hence $\hat{L}^{N} \Psi_{1}\left(\sigma_{L}\right) \geq 0$, if $\tau_{L} \leq \sigma_{L}$. Consider the piecewise linear barrier function

$$
\Psi_{2}\left(x_{i}\right):=\frac{x_{i}}{\sigma_{L}}, 0 \leq x_{i} \leq \sigma_{L} ; \quad \Psi_{2}\left(\sigma_{L}+H\right):=1 .
$$

For $x_{i}<\sigma_{L}, \hat{L}^{N} \Psi_{2}\left(x_{i}\right) \geq 0$ and at the transition point $\sigma_{L}$, using (19),

$$
\hat{L}^{N} \Psi_{2}\left(\sigma_{L}\right)=\frac{1}{h_{L} \sigma_{L}}\left(\mu a h_{L}+\varepsilon\right) \geq \frac{C N}{\theta}(\ln N)^{-2}+C \frac{\mu}{\sqrt{\varepsilon \theta}}(\ln N)^{-1} .
$$

Then we deduce that

$\left|D^{-}\left(W_{L}-w_{L}\right)\left(x_{i}\right)\right| \leq \frac{C}{\sqrt{\varepsilon \theta}} N^{-1} \ln N \Psi_{1}\left(x_{i}\right)+C\left(\frac{\theta}{N} \frac{1}{\varepsilon \theta^{2}}+\frac{N^{-1}}{\sqrt{\varepsilon \theta}}\right) e^{-\rho_{L} \sigma_{L}} \Psi_{2}\left(x_{i}\right)(\ln N)^{2}$. 
For $\tau_{L} \leq x_{i} \leq \sigma_{L}$, noting $e^{-\rho_{L} \tau_{L}} \leq C \rho_{L}^{-2} \leq C \varepsilon \theta$,

$$
\left|D^{-}\left(W_{L}-w_{L}\right)\left(x_{i}\right)\right| \leq \frac{C}{\sqrt{\varepsilon \theta}}\left(N^{-1} \ln N\right) \sqrt{\varepsilon \theta}+C N^{-1}(\ln N)^{2} \leq C N^{-1}(\ln N)^{2} .
$$

(iii) Let us now consider the error $D^{-}\left(W_{R}-w_{R}\right)$ in the right fine mesh subregion $\left(1-\sigma_{R}, 1-\tau_{R}\right)$. Using the truncation error bounds (23) and the exponential bounds in Theorem 1 in the region $\left(1-\sigma_{R}, 1\right)$, we have

$$
\begin{aligned}
\left|\hat{L}^{N} D^{-}\left(W_{R}-w_{R}\right)\left(x_{i}\right)\right| & \leq C N^{-1} \ln N\left(\theta \sqrt{\frac{\theta}{\varepsilon}} e^{-\rho_{R}\left(1-x_{i}\right)}+1\right), x_{i}>1-\sigma_{R}+h_{R} \\
\left|\hat{L}^{N} D^{-}\left(W_{R}-w_{R}\right)\left(x_{i}\right)\right| & \leq C \theta \sqrt{\frac{\theta}{\varepsilon}}\left(1+\sqrt{\frac{\theta}{\varepsilon}} \frac{1}{\ln N}\right) e^{-\rho_{R}\left(1-x_{i}\right)}, \quad x_{i}=1-\sigma_{R}+h_{R} .
\end{aligned}
$$

Consider the barrier function (which is a truncated version of $\Psi_{R}$ defined in (15b))

$$
\Psi_{3}\left(x_{i}\right):=\left(1+0.5 \rho_{R} h_{R}\right)^{-(N-i)}, 1-\sigma_{R}<x_{i} \leq 1 ; \quad \Psi_{3}\left(1-\sigma_{R}\right):=0 .
$$

For $x_{i}>1-\sigma_{R}+h_{R}, \hat{L}^{N} \Psi_{3}\left(x_{i}\right) \geq C \theta e^{-\frac{\rho_{R}}{2}\left(1-x_{i}\right)}$ and $\hat{L}^{N} \Psi_{3}\left(1-\sigma_{R}+h_{R}\right) \geq 0$. This barrier function will be used to deal with the truncation error across the fine mesh region $\left(1-\sigma_{R}+h_{R}, 1\right)$. An additional barrier function is required to manage the larger truncation error at $x_{i}=1-\sigma_{R}+h_{R}$. Consider the step barrier function

$$
\Psi_{4}\left(1-\sigma_{R}\right):=0 ; \quad \Psi_{4}\left(x_{i}\right):=1, \quad 1-\sigma_{R}+h_{R} \leq x_{i} \leq 1 .
$$

For $x_{i}>1-\sigma_{R}+h_{R}, \hat{L}^{N} \Psi_{4}\left(x_{i}\right) \geq 0$ and at the single point $1-\sigma_{R}+h_{R}$, using (19),

$$
\hat{L}^{N} \Psi_{4}\left(1-\sigma_{R}+h_{R}\right) \geq \frac{\mu}{h_{R}}+\frac{\varepsilon}{H h_{R}} \quad\left(\geq C \frac{N \theta}{\ln N}, \text { if } \theta>1\right) .
$$

Then, in the particular case where $\theta>1$, we deduce that

$\left|D^{-}\left(W_{R}-w_{R}\right)\left(x_{i}\right)\right| \leq \frac{C \sqrt{\theta}}{\sqrt{\varepsilon}}\left(N^{-1} \ln N\right) \Psi_{3}\left(x_{i}\right)+C \frac{1}{\theta N} \frac{\theta^{2}}{\varepsilon} e^{-\rho_{R} \sigma_{R}} \Psi_{4}\left(x_{i}\right)+C N^{-1} \ln N$.

For $1-\sigma_{R}<x_{i} \leq 1-\tau_{R}$, we note that on the fine mesh

$$
e^{-\rho_{R} \sigma_{R}} \leq e^{-\rho_{R} \tau_{R}} \leq \rho_{R}^{2} \leq C\left(\frac{\varepsilon}{\theta}\right) \quad \text { and } \quad\left(1+0.5 \rho_{R} h_{R}\right)^{-1} \leq C e^{-\frac{\rho_{R} \tau_{R}}{2}}
$$

Hence, $\left|D^{-}\left(W_{R}-w_{R}\right)\left(x_{i}\right)\right| \leq C N^{-1} \ln N$, if $\theta>1$. When $\theta=1$, we employ an alternative barrier function to $\Psi_{4}\left(x_{i}\right)$ defined as

$$
\Psi_{5}\left(1-\sigma_{R}\right):=1, \quad \Psi_{5}\left(x_{i}\right):=\frac{1-x_{i}}{\sigma_{R}-h_{R}}, 1-\sigma_{R}+h_{R} \leq x_{i} \leq 1 .
$$

Using (19) and the fact that $\frac{b}{a}-\frac{\mu}{\sigma_{R}}>0$, we note that

$$
\hat{L}^{N} \Psi_{5}\left(1-\sigma_{R}+h_{R}\right) \geq C \frac{N}{(\ln N)^{2}} ; \quad \hat{L}^{N} \Psi_{5}\left(x_{i}\right) \geq 0, x_{i}>1-\sigma_{R}+h_{R} .
$$

Then, in the particular case where $\theta=1$, we deduce that

$$
\left|D^{-}\left(W_{R}-w_{R}\right)\left(x_{i}\right)\right| \leq \frac{C \sqrt{\theta}}{\sqrt{\varepsilon}} N^{-1} \ln N \Psi_{3}\left(x_{i}\right)+C N^{-1} \frac{\ln N}{\varepsilon} e^{-\rho_{R} \sigma_{R}} \Psi_{5}\left(x_{i}\right)+C N^{-1} \ln N
$$


For $1-\sigma_{R}<x_{i} \leq 1-\tau_{R}$, we have $e^{-\rho_{R}\left(1-x_{i}\right)} \leq e^{-\rho_{R} \tau_{R}} \leq C \varepsilon$. Hence,

$$
\left|D^{-}\left(W_{R}-w_{R}\right)\left(x_{i}\right)\right| \leq C N^{-1} \ln N+C N^{-1} \ln N \frac{\varepsilon}{\varepsilon} \leq C N^{-1} \ln N, \quad \text { if } \theta=1 .
$$

(iv) We complete the argument, by dealing with the regular component. In the case of $\theta=1$, note the bound (42) for the regular component. Let us consider the regular component in the case of $\theta>1$. Note that $\left\|L^{N}(v-V)\right\| \leq C(\varepsilon+\mu) N^{-1}$ and so

$$
\|V-v\| \leq C N^{-1} \mu, \quad \theta>1 .
$$

Note that we can confine the discussion to the mesh points in the region $\left(1-\sigma_{R}, 1-\tau_{R}\right)$. Within the fine mesh region $\left(1-\sigma_{R}, 1\right)$, the error in the flux satisfies the first order problem

$$
-\frac{\varepsilon}{h_{R}}\left(\mathscr{V}_{i+1}^{-}-\mathscr{V}_{i}^{-}\right)+\mu a\left(x_{i}\right) \mathscr{V}_{i}^{-}=\hat{\mathscr{T}}_{i}, \quad\left|\mathscr{V}_{N}^{-}\right| \leq C \frac{\mu}{\varepsilon} N^{-1}
$$

where $\hat{\mathscr{T}}_{i}:=L^{N}(V-v)\left(x_{i}\right)-b\left(x_{i}\right)(V-v)\left(x_{i}\right)$. Note further that

$$
\|\hat{\mathscr{T}}\| \leq C N^{-1}(\varepsilon+\mu) \leq C \mu N^{-1}
$$

Thus, with $\rho:=\frac{\alpha \mu h_{R}}{\varepsilon} \leq C N^{-1} \ln N$, we have

$$
\left|\mathscr{V}_{i}^{-}\right|=\left(1+\frac{\mu h_{R}}{\varepsilon} a\left(x_{i}\right)\right)^{-1}\left|\frac{h_{R}}{\varepsilon} \hat{\mathscr{T}}_{i}+\mathscr{V}_{i+1}^{-}\right| \leq C(1+\rho)^{-1}\left(\frac{\rho}{\mu}\|\hat{\mathscr{T}}\|+\left|\mathscr{V}_{i+1}^{-}\right|\right)
$$

We have the following estimate at $x_{i}$ (within the fine mesh where $(1+\rho)^{-1} \leq C e^{-\rho / 2}$ for $N$ sufficiently large)

$$
\begin{aligned}
\left|\mathscr{V}_{i}^{-}\right| & \leq(1+\rho)^{-1} \frac{\rho}{\mu}\|\hat{\mathscr{T}}\| \frac{1-(1+\rho)^{-(N-i)}}{1-(1+\rho)^{-1}}+C(1+\rho)^{-(N-i)}\left|\mathscr{V}_{N}^{-}\right| \\
& \leq C N^{-1}+C \frac{\mu N^{-1}}{\varepsilon}(1+\rho)^{-(N-i)} \leq C N^{-1}+C \frac{\mu N^{-1}}{\varepsilon} e^{-\frac{\alpha \mu}{2 \varepsilon}\left(1-x_{i}\right)}
\end{aligned}
$$

Hence, for $1-\sigma_{R}<x_{i} \leq 1-\tau_{R},\left|\mathscr{V}_{i}^{-}\right| \leq C N^{-1}$. 